\title{
QUADRATICALLY PINCHED HYPERSURFACES OF THE SPHERE VIA MEAN CURVATURE FLOW WITH SURGERY
}

\author{
MAT LANGFORD AND HUY THE NGUYEN
}

\begin{abstract}
We study mean curvature flow in $\mathbb{S}_{K}^{n+1}$, the round sphere of sectional curvature $K>0$, under the quadratic curvature pinching condition $|A|^{2}<\frac{1}{n-2} H^{2}+4 K$ when $n \geq 4$ and $|A|^{2}<\frac{3}{5} H^{2}+\frac{8}{3} K$ when $n=3$. This condition is related to a famous theorem of Simons [28, which states that the only minimal hypersurfaces satisfying $|A|^{2}<n K$ are the totally geodesic hyperspheres. It is related to but distinct from the "two-convexity" condition studied in [10,21. Notably, in contrast to two-convexity, it allows the mean curvature to change sign. We show that the pinching condition is preserved by mean curvature flow, and obtain a "cylindrical" estimate and corresponding pointwise derivative estimates for the curvature. As a result, we find that the flow becomes either uniformly convex or quantitatively cylindrical in regions of high curvature. This allows us to apply the surgery apparatus developed by Huisken and Sinestrari [21] (cf. [16]). We conclude that any smoothly, properly, isometrically immersed hypersurface $\mathcal{M}$ of $\mathbb{S}_{K}^{n+1}$ satisfying the pinching condition is diffeomorphic to $\mathbb{S}^{n}$ or the connected sum of a finite number of copies of $\mathbb{S}^{1} \times \mathbb{S}^{n-1}$. If $\mathcal{M}$ is embedded, then it bounds a 1-handlebody. The results are sharp when $n \geq 4$.
\end{abstract}

\section{Contents}

1. Introduction

Acknowledgements

2. Preliminaries

3. Preserved curvature conditions

4. The key estimates for smooth flows

5. The key estimates for surgically modified flows

6. Existence of terminating surgically modified flows

References

2000 Mathematics Subject Classification. Primary 53C44. 


\section{INTRODUCTION}

Beginning in the late 1960's, Simons [28 and others [1, 13, 14, 27] obtained rigidity theorems for minimal and constant mean curvature hypersurfaces in the sphere under certain bounds on the second fundamental form (depending on the dimension and the value of the mean curvature). The results are obtained by exploiting Simons'identity, a Bochner-like formula which relates the Hessian of the mean curvature (which vanishes for a constant mean curvature hypersurface) to the Laplacian of the second fundamental form. Simons' theorem, for example, states that the only minimal hypersurfaces of $\mathbb{S}^{n+1}$ satisfying $|A|^{2} \leq n$, where $A$ denotes the second fundamental form, are the totally geodesic hyperspheres (which satisfy $|A|^{2} \equiv 0$ ) and the Clifford hypersurfaces (which satisfy $|A|^{2}=n$ ).

Such results can be improved upon using curvature flows, which remove the constant mean curvature restriction. Indeed, Huisken [20] showed that, under mean curvature flow, hypersurfaces of the sphere $\mathbb{S}_{K}^{n+1}$ of sectional curvature 1$], n \geq 2$, satisfying the quadratic curvature pinching condition

$$
\begin{cases}|A|^{2}<\frac{1}{n-1} H^{2}+2 K & \text { if } n \geq 3 \\ |A|^{2}<\frac{3}{4} H^{2}+\frac{4}{3} K & \text { if } n=2\end{cases}
$$

shrink, preserving the inequality, either to a "round" point in finite time or to a totally geodesic hypersphere in infinite time. In case $n \geq 3$, this behaviour is sharp in the sense that there exist hypersurfaces of the form $\mathbb{S}^{1}(r) \times \mathbb{S}^{n-1}(s), r^{2}+s^{2}=1$, on which $|A|^{2}-\frac{1}{n-1} H^{2}$ can be made arbitrarily close to 2 . Andrews [2] obtained a sharper result when $n=2$ : he showed that, under a different (fully nonlinear) curvature flow, positive sectional curvature (which is equivalent to the inequality $\left.|A|^{2}<H^{2}+2\right)$ is preserved, and solutions converge either to round points in finite time, or totally geodesic spheres in infinite time.

We will develop these results further by allowing a weaker curvature pinching condition. Namely, we study, for $n \geq 3$, hypersurfaces of $\mathbb{S}_{K}^{n+1}$ satisfying

$$
\begin{cases}|A|^{2}<\frac{1}{n-2} H^{2}+4 K & \text { if } n \geq 4 \\ |A|^{2}<\frac{3}{5} H^{2}+\frac{8}{3} K & \text { if } n=3\end{cases}
$$

The analysis is much more complicated under the weaker condition (1.2), since we can no longer expect solutions to shrink to a round

\footnotetext{
${ }^{1}$ We find it convenient to work without normalizing the curvature $K$, as it serves as a natural scale parameter.
} 
point at a finite time singularity — further singularities and topologies are possible. The purpose of the pinching condition (1.2) is to ensure that the only additional singularities are (possibly degenerate) "neck-pinch" singularities. In this respect, our results are sharp (when $n \geq 4)$, since there exist hypersurfaces of $\mathbb{S}^{n+1}$ the form $\mathbb{S}^{2}(r) \times \mathbb{S}^{n-2}(s)$, $r^{2}+s^{2}=1$, on which $|A|^{2}-\frac{1}{n-2} H^{2}$ can be made arbitrarily close to 4 . Once this is established, we are able to make use of the robust surgery construction of Huisken and Sinestrari [21], which allows us to replace the singular neck regions by almost spherical caps, and thereby continue the flow (cf. [10]). Since the estimates hold in the presence of surgeries, with constants that do not depend on the maximal time of existence, we find, after a finite number of surgeries, that the initial hypersurface has decomposed into a finite number of components, each of which is either a "small" $\mathbb{S}^{n}$, the Cartesian product of $\mathbb{S}^{1}$ with a "small" $\mathbb{S}^{n-1}$, or a "large" $\mathbb{S}^{n}$. As a consequence, we obtain a classification of diffeomorphism types for hypersurfaces satisfying the pinching condition.

Theorem 1.1. Every properly, isometrically immersed hypersurface $X: \mathcal{M} \rightarrow \mathbb{S}_{K}^{n+1}$ of $\mathbb{S}_{K}^{n+1}$ satisfying (1.2) is diffeomorphic either to $\mathbb{S}^{n}$ or to a connected sum of finitely many copies of $\mathbb{S}^{1} \times \mathbb{S}^{n-1}$. Indeed, there exists a 1-handlebody $\Omega$ and an immersion $\bar{X}: \Omega \rightarrow \mathbb{S}_{K}^{n+1}$ such that $\partial \Omega$ is diffeomorphic to $\mathcal{M}$ and $\left.\bar{X}\right|_{\partial \Omega}=X$. If $X$ is an embedding, then so is $\bar{X}$.

Our arguments follow those of Huisken and Sinestrari [21] - however we first establish a cylindrical estimate (Theorem 4.1) by way of Stampacchia iteration, and then use this to obtain pointwise derivative estimates for the curvature using the maximum principle (Theorems 4.8 and 4.11). These estimates allow us to apply, virtually unmodified, the Huisken-Sinestrari surgery algorithm after pulling the flow locally up to the tangent space to $\mathbb{S}_{K}^{n+1}$. In particular, we do not use positive mean curvature and convexity estimates follow as a consequence of the cylindrical estimates.

The cylindrical estimate may be viewed as a partial generalization of Simons' famous theorem mentioned above. It also implies a new rigidity theorem for ancient solutions to mean curvature flow in the sphere (Corollary 4.4). The key to proving it is a Poincaré-type inequality for $W^{2,2}$-functions supported away from "cylindrical" points of the hypersurface (Proposition 2.2).

We also obtain novel noncollapsing estimates (Corollary 3.3) which are preserved by the surgery algorithm (see \$5.1). These are not actually required for the proof of Theorem 1.1, but we include them 
here as they may be of some use in obtaining further applications (cf. [11, 12, 24]).

The work of Huisken and Sinestrari was generalized in a different direction by Brendle and Huisken [10] who, building upon earlier work of Andrews [3], studied the evolution (with surgeries) by a certain fully nonlinear flow of hypersurfaces in compact Riemannian ambient spaces satisfying the two-convexity condition

$$
\lambda_{1}+\lambda_{2}>2 \sqrt{-K},
$$

where $\lambda_{1} \leq \lambda_{2} \leq \cdots \leq \lambda_{n}$ are the principal curvatures of the hypersurface and $K \leq 0$ is a lower bound for the sectional curvatures of the ambient space. For hypersurfaces of the sphere, this becomes ordinary two-convexity,

$$
\lambda_{1}+\lambda_{2}>0 .
$$

A consequence of their work is a version of Theorem 1.1 for hypersurfaces with the quadratic pinching condition (1.2) replaced by twoconvexity. Note that two-convexity is neither stronger nor weaker than the quadratic pinching condition (1.2). Indeed, unlike two-convexity, the quadratic condition (1.2) is invariant under orientation reversal, and therefore allows the mean curvature to change sign. Moreover, the Brendle-Huisken approach holds only for embedded hypersurfaces, since they require noncollapsing estimates (see [4, 7, 9]) to obtain a gradient estimate for the curvature (cf. [15]).

\section{ACKNOWLEDGEMENTS}

M. Langford was supported by an Alexander von Humboldt fellowship and an Australian Research Council DECRA fellowship.

H. T. Nguyen was supported by the EPSRC grant EP/S012907/1.

\section{Preliminaries}

2.1. Hypersurfaces of $\mathbb{S}_{K}^{n+1}$. Here we recall the fundamental identities for immersed hypersurfaces $X: \mathcal{M} \rightarrow \mathbb{S}_{K}^{n+1}$ of the sphere $\mathbb{S}_{K}^{n+1}$ of sectional curvature $K>0$. First, recall the Gauss equation

$$
\mathrm{Rm}_{i j k l}=A_{i k} A_{j l}-A_{i l} A_{j k}+K\left(g_{i k} g_{j l}-g_{i l} g_{j k}\right),
$$

where $A_{i j}^{2}:=A_{i}^{p} A_{p j}$, and its traces

$$
\mathrm{Rc}_{i k}=\left(H A_{i k}-A_{i k}^{2}\right)+(n-1) K g_{i k}
$$

and

$$
\mathrm{Sc}=H^{2}-|A|^{2}+n(n-1) K \text {. }
$$


The Codazzi equation,

$$
\nabla_{k} A_{i j}=\nabla_{i} A_{k j}
$$

implies that the covariant differential $\nabla A$ of the second fundamental form is totally symmetric.

Combining the Gauss and Codazzi equations yields Simons' identity

$$
\nabla_{(i} \nabla_{j)} A_{k l}-\nabla_{(k} \nabla_{l)} A_{i j}=A_{i j} A_{k l}^{2}-A_{k l} A_{i j}^{2}+K\left(g_{i j} A_{k l}-g_{k l} A_{i j}\right)
$$

where brackets indicate symmetrization about the enclosed components, and its trace

$$
\Delta A_{i j}=\nabla_{i} \nabla_{j} H+H A_{i j}^{2}-|A|^{2} A_{i j}-K\left(H g_{i j}-n A_{i j}\right) .
$$

By splitting $\nabla A$ into is trace and trace-free parts, we obtain the Kato inequality

$$
|\nabla A|^{2} \geq \frac{3}{n+2}|\nabla H|^{2}
$$

2.2. Mean curvature flow in $\mathbb{S}_{K}^{n+1}$. Next, we recall the fundamental identities for a family of hypersurfaces $X: \mathcal{M} \times I \rightarrow \mathbb{S}_{K}^{n+1}$ evolving by mean curvature flow. We make use of the time-dependent connection of Andrews and Baker [5], which differentiates time-dependent tangent vector fields $V$ on $\mathcal{M}$ in space-time directions $\xi \in T(\mathcal{M} \times I)$ in the obvious way:

$$
d X\left(\nabla_{\xi} V\right):=\left(D_{\xi}[d X(V)]\right)^{\top},
$$

where $D$ is the pullback to $\mathcal{M} \times I$ of the ambient connection, $d X$ is the differential of $X$ and $(\cdot)^{\top}$ the projection onto $d X(T \mathcal{M})$. Observe that

$$
\nabla_{\xi} V=\left[\partial_{t}, V\right]-H A(V),
$$

where $[\cdot, \cdot]$ denotes the Lie bracket and we conflate the second fundamental form with the Weingarten map.

Note that $\nabla_{\xi}$ agrees with the Levi-Civita covariant derivative on the spatial tangent bundle $\{\xi \in T(\mathcal{M} \times I): d t(\xi)=0\}$ (which we conflate with $T \mathcal{M}$ ) when $\xi$ has no $\partial_{t}$ component, where $\partial_{t}$ is the canonical tangent vector field to $I$. The main advantage of working with the time-dependent connection (as opposed to the Lie derivative) is that the induced metric tensor $g$ is $\nabla_{t}$-parallel:

$$
\nabla_{t} g=0
$$

where $\nabla_{t}:=\nabla_{\partial_{t}}$. 
Denote by ${ }^{X} \overline{\mathrm{Rm}}$ the curvature tensor of the pullback connection ${ }^{X} D$ and let $U$ and $V$ be any pair of time-dependent tangent vector fields. Observe, on the one hand, that

$$
\begin{aligned}
{ }^{X} \overline{\operatorname{Rm}}\left(\partial_{t}, U\right) V= & d X\left[\operatorname{Rm}\left(\partial_{t}, U\right) V+A(U, V) \nabla H-\nabla_{V} H A(U)\right] \\
& +\left[\nabla_{t} A(U, V)-\nabla_{U} \nabla_{V} H-H A^{2}(U, V)\right] \nu,
\end{aligned}
$$

where we conflate $\nabla H$ with the gradient of $H$. On the other hand,

$$
\begin{aligned}
{ }^{X} \overline{\operatorname{Rm}}\left(\partial_{t}, U\right) V & =\overline{\operatorname{Rm}}\left(d X\left(\partial_{t}\right), d X(U)\right)(d X(V)) \\
& =-H \overline{\operatorname{Rm}}(\nu, d X(U)) d X(V) \\
& =H K g(U, V) \nu .
\end{aligned}
$$

Resolving (2.6) into tangential and normal components, we obtain the "temporal" Gauss-Codazzi equations

$$
\operatorname{Rm}\left(\partial_{t}, U\right) V=\nabla_{V} H A(U)-A(U, V) \nabla H
$$

and

$$
\nabla_{t} A=\nabla^{2} H+H A^{2}+K H g
$$

respectively, where in (2.8) both sides are understood as tensors on the spatial tangent bundle.

Combining the Codazzi identity (2.8) with the contracted Simons identity (2.3) yields an evolution equation for the second fundamental form:

$$
\left(\nabla_{t}-\Delta\right) A=\left(|A|^{2}+n K\right) A-2 n K\left(A-\frac{1}{n} H g\right),
$$

where $\Delta$ is the spatial Laplacian. Tracing yields

$$
\left(\partial_{t}-\Delta\right) H=\left(|A|^{2}+n K\right) H,
$$

which immediately yields

$$
\left(\partial_{t}-\Delta\right) H^{2}=-2|\nabla H|^{2}+2\left(|A|^{2}+n K\right) H^{2} .
$$

Since $g$ is $\nabla_{t}$-parallel, (2.9) immediately yields

$$
\begin{aligned}
\left(\partial_{t}-\Delta\right)|A|^{2}= & -2|\nabla A|^{2}+2\left(|A|^{2}+n K\right)|A|^{2} \\
& -4 n K\left(|A|^{2}-\frac{1}{n} H^{2}\right)
\end{aligned}
$$

where $\nabla A$ is the spatial covariant differential of $A$.

Given tensor fields $S$ and $T$, we denote by $S * T$ any tensor field resulting from linear combinations of metric contractions of $S \otimes T$. By (2.5) and (2.7),

$$
\nabla_{t}(\nabla T)=\nabla\left(\nabla_{t} T\right)+A * A * \nabla T+A * \nabla A * T .
$$


By (2.1),

$$
\Delta(\nabla T)=\nabla(\Delta T)+A * A * \nabla T+K * \nabla T+A * \nabla A * T .
$$

Thus,

$$
\begin{aligned}
\left(\nabla_{t}-\Delta\right)(\nabla A) & =\nabla\left[\left(\nabla_{t}-\Delta\right) A\right]+A * A * \nabla A+K * \nabla A \\
& =A * A * \nabla A+K * \nabla A,
\end{aligned}
$$

and hence, by Young's inequality,

$$
\left(\partial_{t}-\Delta\right)|\nabla A|^{2} \leq-2\left|\nabla^{2} A\right|^{2}+c_{n}\left(|A|^{2}+n K\right)|\nabla A|^{2}
$$

where $c_{n}$ is a constant that depends only on $n$.

Similarly,

$$
\left(\nabla_{t}-\Delta\right)\left(\nabla^{2} A\right)=A * A * \nabla^{2} A+A * \nabla A * \nabla A+K * \nabla^{2} A,
$$

and hence

$$
\left(\partial_{t}-\Delta\right)\left|\nabla^{2} A\right|^{2} \leq-2\left|\nabla^{3} A\right|^{2}+c_{n}\left[\left(|A|^{2}+n K\right)\left|\nabla^{2} A\right|^{2}+|\nabla A|^{4}\right],
$$

where $c_{n}$ is a constant that depends only on $n$.

Similar inequalities hold for higher derivatives of $A$ since, by a straightforward induction argument,

$$
\left(\nabla_{t}-\Delta\right)\left(\nabla^{m} A\right)=K * \nabla^{m} A+\sum_{i+j+k=m} \nabla^{i} A * \nabla^{j} A * \nabla^{k} A
$$

The following "Bernstein estimates" are a standard application of the "rough" evolution equations (2.15). For a proof in the Euclidean case (which carries over with minor modifications) see, for example, 6 , Theorem 6.24].

Proposition 2.1 (Bernstein estimates). Let $X: \mathcal{M} \times\left[0, \lambda K^{-1}\right] \rightarrow \mathbb{S}_{K}^{n+1}$ be a solution to mean curvature flow. If

$$
\max _{\mathcal{M} \times\left[0, \lambda K^{-1}\right]}|A|^{2} \leq \Lambda_{0} K
$$

then

$$
t^{m}\left|\nabla^{m} A\right|^{2} \leq \Lambda_{m} K
$$

where $\Lambda_{m}$ depends only on $n, m, \lambda$ and $\Lambda_{0}$.

2.3. A Poincaré-type inequality. The following Poincaré-type inequality (cf. [18, 5.4 Lemma]) is crucial to obtaining the cylindrical estimate (via Stampacchia iteration) in Section 4.1.

Proposition 2.2. Given $n \geq 3, \alpha \in(0,1)$ and $\eta \in\left(0, \frac{1}{n-2+\alpha}-\frac{1}{n-1}\right)$ there exists $\gamma=\gamma(n, \alpha, \eta)>0$ with the following property: Let $X$ : 
$\mathcal{M}^{n} \rightarrow \mathbb{S}_{K}^{n+1}$ be a smoothly immersed hypersurface and let $u \in W^{1,2}(\mathcal{M})$ be a function satisfying spt $u \subset U_{\alpha, \eta}$, where, introducing the functions

$$
f_{1, \eta}:=|A|^{2}-\left(\frac{1}{n-1}+\eta\right) H^{2}
$$

and

$$
g_{2, \alpha}:=|A|^{2}-\frac{1}{n-2+\alpha} H^{2}-2(2-\alpha) K,
$$

the "acylindrical" set $U_{\alpha, \eta} \subset \mathcal{M}$ is defined by

$$
U_{\alpha, \eta}:=\left\{x \in \mathcal{M}: f_{1, \eta}(x) \geq 0 \geq g_{2, \alpha}(x)\right\} .
$$

For any $r \geq 1$,

$$
\gamma \int u^{2} W d \mu \leq \int u^{2}\left(r^{-1} \frac{|\nabla u|^{2}}{u^{2}}+r \frac{|\nabla A|^{2}}{W}+K\right) d \mu,
$$

where

$$
W:=\left(\frac{1}{n-2+\alpha}-\frac{1}{n-1}-\eta+\frac{\alpha}{2 n(n-1)}\right) H^{2}+2(2-\alpha) K .
$$

Proof. By a straightforward scaling argument, it suffices to prove the claim when $K=1$. By a standard approximation argument, we may assume that $u$ is smooth.

Recall Simons' identity

$$
\nabla_{(i} \nabla_{j)} A_{k l}-\nabla_{(k} \nabla_{l)} A_{i j}=\mathrm{C}_{i j k l},
$$

where the brackets denote symmetrization and

$$
\mathrm{C}:=A \otimes A^{2}-A^{2} \otimes A+(g \otimes A-A \otimes g) .
$$

We claim that

$$
\gamma W^{3} \leq|\mathrm{C}|^{2}+1 \quad \text { in } \quad U_{\alpha, \eta}
$$

on any immersed hypersurface $X: \mathcal{M}^{n} \rightarrow \mathbb{S}^{n+1}$ for some positive $\gamma=\gamma(n, \alpha, \eta)$. Indeed, if this is not the case, then there is a sequence $\left\{\vec{\lambda}^{k}\right\}_{k \in \mathbb{N}}$ of vectors $\vec{\lambda}^{k} \in \mathbb{R}^{n}$ (corresponding to principal curvatures of a sequence of hypersurfaces) satisfying

$$
f_{1, \eta}\left(\vec{\lambda}^{k}\right):=\left|\vec{\lambda}^{k}\right|^{2}-\frac{1}{n-1} \operatorname{tr}\left(\vec{\lambda}^{k}\right)^{2}-\eta \operatorname{tr}\left(\vec{\lambda}^{k}\right)^{2} \geq 0
$$

and

$$
g_{2, k}\left(\vec{\lambda}^{k}\right):=\left|\vec{\lambda}^{k}\right|^{2}-\frac{1}{n-2+k} \operatorname{tr}\left(\vec{\lambda}^{k}\right)^{2}-2(2-k) \leq 0,
$$

where $\operatorname{tr}(\vec{\lambda}):=\sum_{i=1}^{n} \lambda_{i}$, but

$$
\frac{\left|\mathrm{C}\left(\vec{\lambda}^{k}\right)\right|^{2}+1}{W^{3}\left(\vec{\lambda}^{k}\right)} \rightarrow 0
$$


as $k \rightarrow \infty$, where

$$
|\mathrm{C}(\vec{\lambda})|^{2}:=\sum_{i, j=1}^{n}\left(\lambda_{j}-\lambda_{i}\right)^{2}\left(\lambda_{i} \lambda_{j}+1\right)^{2}
$$

and

$$
W(\vec{\lambda}):=\left(\frac{1}{n-2+\alpha}-\frac{1}{n-1}-\eta+\frac{\alpha}{2 n(n-1)}\right) \operatorname{tr}(\vec{\lambda})^{2}+2(2-\alpha) .
$$

Set $r_{k}^{2}:=W\left(\vec{\lambda}^{k}\right)^{-1} \rightarrow 0$ and $\hat{\lambda}^{k}:=r_{k} \vec{\lambda}^{k}$. Observe that

$$
\left|\hat{\lambda}^{k}\right| \leq \frac{2 n(n-1)}{\alpha(n-2)}=: c(n, \alpha)
$$

and hence, up to a subsequence, $\hat{\lambda}^{k} \rightarrow \hat{\lambda} \in \mathbb{R}^{n}$. Computing

$$
\left(\left|\hat{\lambda}^{k}\right|^{2}-\frac{1}{n-1} \operatorname{tr}\left(\hat{\lambda}^{k}\right)^{2}\right)-\eta \operatorname{tr}\left(\hat{\lambda}^{k}\right)^{2}=r_{k}^{2} f_{1, \eta}\left(\vec{\lambda}^{k}\right) \geq 0
$$

and

$$
\left(\left|\hat{\lambda}^{k}\right|^{2}-\frac{1}{n-2+\alpha} \operatorname{tr}\left(\hat{\lambda}^{k}\right)^{2}\right)-2(2-\alpha) r_{k}^{2}=r_{k}^{2} g_{2, \alpha}\left(\vec{\lambda}^{k}\right) \leq 0
$$

we find

$$
\left(|\hat{\lambda}|^{2}-\frac{1}{n-1} \operatorname{tr}(\hat{\lambda})^{2}\right) \geq \eta \operatorname{tr}(\hat{\lambda})^{2}
$$

and

$$
\left(|\hat{\lambda}|^{2}-\frac{1}{n-2+\alpha} \operatorname{tr}(\hat{\lambda})^{2}\right) \leq 0 .
$$

On the other hand,

$$
\sum_{i, j=1}^{n}\left(\hat{\lambda}_{i}^{k} \hat{\lambda}_{j}^{k}\left(\hat{\lambda}_{j}^{k}-\hat{\lambda}_{i}^{k}\right)\right)^{2}+2 r_{k}^{2} \hat{\lambda}_{i}^{k} \hat{\lambda}_{j}^{k}\left(\hat{\lambda}_{j}^{k}-\hat{\lambda}_{i}^{k}\right)^{2}+r_{k}^{4}\left(\hat{\lambda}_{j}^{k}-\hat{\lambda}_{i}^{k}\right)^{2}=r_{k}^{6}\left|\mathrm{C}\left(\vec{\lambda}^{k}\right)\right|^{2}
$$

so that

$$
\sum_{i, j=1}^{n}\left(\hat{\lambda}_{i} \hat{\lambda}_{j}\left(\hat{\lambda}_{j}-\hat{\lambda}_{i}\right)\right)^{2}=0
$$

Together, (2.17), (2.18) and (2.19) are in contradiction: (2.19) implies that $\hat{\lambda}$ has a null component of multiplicity $m$ and a non-zero component, $\kappa$ say, of multiplicity $n-m$. The inequalities (2.17) and (2.18) then yield

$$
\left(n-m-\frac{(n-m)^{2}}{n-1}\right) \kappa^{2} \geq \eta(n-m)^{2} \kappa^{2}>0
$$


and

$$
\left(n-m-\frac{(n-m)^{2}}{n-2+\alpha}\right) \kappa^{2} \leq 0
$$

which together imply that $m \in(1,2-\alpha]$, which is impossible. This proves (2.16).

Using (2.16), we can estimate

$$
\begin{aligned}
\gamma \int u^{2} W d \mu & \leq \int \frac{u^{2}}{W^{2}}\left(|\mathrm{C}|^{2}+1\right) d \mu \\
& =\int \frac{u^{2}}{W^{2}}\left(\mathrm{C} * \nabla^{2} A+1\right) d \mu \\
& =\int \frac{u^{2}}{W^{2}}\left(\frac{\nabla u}{u} * \mathrm{C}+\frac{\nabla W}{W} * \mathrm{C}+\nabla \mathrm{C}\right) * \nabla A d \mu+\int \frac{u^{2}}{W^{2}} d \mu \\
& \leq C\left[\int \frac{u^{2}}{W^{2}}\left(W^{\frac{3}{2}} \frac{|\nabla u|}{u}+W^{\frac{1}{2}}|\nabla W|+W|\nabla A|\right)|\nabla A| d \mu\right. \\
& \leq C\left[\int u^{2}\left(\frac{|\nabla u|}{u}+\frac{|\nabla A|}{W^{\frac{1}{2}}}\right) \frac{|\nabla A|}{W^{\frac{1}{2}}} d \mu+\int u^{2} d \mu\right],
\end{aligned}
$$

where $C$ denotes any constant which depends only on $n, \alpha$ and $\eta$. The claim now follows from Young's inequality.

2.4. The Sobolev inequality. We shall also require the following Sobolev inequality for the Stampacchia iteration argument in Section 4.1. It may be obtained from [17, Theorem 2.1] (cf. [23]) by the substitution $u \mapsto u^{2}$.

Theorem 2.3. Let $X: \mathcal{M} \rightarrow \mathbb{S}_{K}^{n+1}, n \geq 3$, be a hypersurface of the sphere of sectional curvature $K>0$ and let $u: \mathcal{M} \rightarrow \mathbb{R} a W^{1,2}$ be function. If

$$
\mu(\operatorname{spt}(u)) \leq \frac{\omega_{n}}{n+1} K^{-\frac{n}{2}}
$$

then

$$
\left(\int u^{2^{*}} d \mu\right)^{\frac{1}{2^{*}}} \leq c_{n} \int\left(|\nabla u|^{2}+u^{2} H^{2}\right) d \mu,
$$

where $q^{*}:=\frac{n q}{n-q}$, and $c_{n}$ is a constant that depends only on $n$. 


\section{Preserved Curvature Conditions}

3.1. Quadratic curvature condition. If the strict quadratic curvature inequality (1.2) holds on a hypersurface of $\mathbb{S}_{K}^{n+1}, n \geq 3$, then we can find some $\alpha>0$ such that

$$
|A|^{2} \leq \frac{1}{n-2+\alpha} H^{2}+2(2-\alpha) K .
$$

This inequality is preserved under mean curvature flow when $n \geq 3$ (note that $\alpha>\frac{2}{3}$ when $n=3$ ).

Proposition 3.1 (Cf. [19, 1.4 Lemma]). Let $X: \mathcal{M}^{n} \times[0, T) \rightarrow \mathbb{S}_{K}^{n+1}$, $n \geq 3$, be a solution to mean curvature flow such that (3.1) holds on $\mathcal{M}^{n} \times\{0\}$ for some $\alpha \in(0,1)$. If $n \geq 4$, or if $n=3$ and $\alpha \geq \frac{2}{3}$, then (3.1) holds on $\mathcal{M}^{n} \times\{t\}$ for all $t \in[0, T)$.

Proof. Suppose that (3.1) holds on $\mathcal{M} \times\{0\}$ for some $\alpha \in(0,1)$ when $n \geq 4$ or some $\alpha \in\left(\frac{2}{3}, 1\right)$ when $n=3$. Setting

$$
a_{n}:=\frac{1}{n-2+\alpha} \text { and } b_{n}:=2(2-\alpha),
$$

we compute, using (2.11) and (2.12),

$$
\begin{aligned}
\left(\partial_{t}-\Delta\right)\left(|A|^{2}-a_{n} H^{2}\right)= & -2\left(|\nabla A|^{2}-a_{n}|\nabla H|^{2}\right)+2 b_{n} K\left(|A|^{2}+n K\right) \\
& +2\left(|A|^{2}-a_{n} H^{2}-b_{n} K\right)\left(|A|^{2}+n K\right) \\
& -4 n K\left(|A|^{2}-\frac{1}{n} H^{2}\right) .
\end{aligned}
$$

Since $\frac{2}{2 n-b_{n}}=a_{n}$ and $\frac{n}{2 n-b_{n}} \leq 1$, we can estimate

$$
\begin{array}{rl}
2 b_{n} K\left(|A|^{2}+n K\right)-4 & K\left(|A|^{2}-\frac{1}{n} H^{2}\right) \\
& =2 K\left(\left(b_{n}-2 n\right)|A|^{2}+2 H^{2}+b_{n} n K\right) \\
& =-2 K\left(2 n-b_{n}\right)\left(|A|^{2}-\frac{2}{2 n-b_{n}} H^{2}-\frac{n b_{n}}{2 n-b_{n}} K\right) \\
\leq & -2 K\left(2 n-b_{n}\right)\left(|A|^{2}-a_{n} H^{2}-b_{n} K\right) .
\end{array}
$$

Estimating $\frac{2}{2 n-b_{n}} \leq \frac{3}{n+2}$ and applying (2.4), we arrive at

$$
\begin{aligned}
\left(\partial_{t}-\Delta\right)\left(|A|^{2}-a_{n} H^{2}\right. & \left.-b_{n} K\right) \\
& \leq 2\left(|A|^{2}+\left(b_{n}-n\right) K\right)\left(|A|^{2}-a_{n} H^{2}-b_{n} K\right) .
\end{aligned}
$$

The claim now follows from the maximum principle. 
3.2. Rigidity. The quadratic curvature condition (1.2) is optimal for cylindrical estimates and connected sum theorems in dimensions $n \geq$ 4. Indeed, consider the hypersurfaces $M^{k, n-k}(r, s)=\mathbb{S}^{k}(r) \times \mathbb{S}^{n-k}(s)$, $r^{2}+s^{2}=1$, of $\mathbb{S}^{n+1}$, where $\mathbb{S}^{k}(r)$ is the $k$ dimensional sphere of radius $r$. The second fundamental forms have eigenvalues $\lambda$, with multiplicity $k$, and $\mu$, with multiplicity $n-k$, such that $\lambda \mu=-1$.

Consider the case $k=2$. In this case,

$$
|A|^{2}=\frac{2 s^{4}+(n-2) r^{4}}{r^{2} s^{2}}
$$

and

$$
H=\frac{(n-2) r^{2}-2 s^{2}}{r s}
$$

so that

$$
H^{2}=\frac{(n-2)^{2} r^{4}+4 s^{4}-4(n-2) r^{2} s^{2}}{r^{2} s^{2}},
$$

which then yields

$$
|A|^{2}-\frac{1}{n-2} H^{2}-4=\frac{2(n-4)}{(n-2)} \frac{s^{2}}{r^{2}} .
$$

Thus, in every dimension $n \geq 4$, we can find, for any $\varepsilon>0$, a hypersurface of the topological type $\mathbb{S}^{2} \times \mathbb{S}^{n-2}$ satisfying

$$
|A|^{2}-\frac{1}{n-2} H^{2}-4 \leq \varepsilon \text {. }
$$

So the quadratic bound (1.2) in Theorem 1.1 is the best that can be achieved when $n \geq 4$.

3.3. Inscribed/exscribed curvature pinching. We now present a noncollapsing estimate for mean curvature flow under the quadratic pinching condition (1.2). As mentioned in the introduction, this will not actually be required to obtain the main result (Theorem 1.11).

As in [7, 9], we define the inscribed and exscribed curvatures $\bar{k}$ and $\underline{k}$ of an embedded hypersurface $\mathcal{M} \hookrightarrow \mathbb{S}_{K}^{n+1}$ by

$$
\bar{k}(p):=\sup _{q \neq p} \frac{2\langle p-q, \nu(p)\rangle}{\|p-q\|^{2}} \text { and } \underline{k}(p):=\inf _{q \neq p} \frac{2\langle p-q, \nu(p)\rangle}{\|p-q\|^{2}},
$$

respectively, where the inner product and norm are those of $\mathbb{R}^{n+2}$. Note that, under orientation reversal, $\bar{k} \mapsto-\underline{k}$ and $\underline{k} \mapsto-\bar{k}$. Observe also that

$$
\bar{k}(p) \geq \limsup _{q \rightarrow p} \frac{2\langle p-q, \nu(p)\rangle}{\|p-q\|^{2}}=\lambda_{n}
$$


and

$$
\underline{k}(p) \leq \liminf _{q \rightarrow p} \frac{2\langle p-q, \nu(p)\rangle}{\|p-q\|^{2}}=\lambda_{1}
$$

In particular,

$$
\bar{k} \geq \frac{1}{n} H \text { and } \underline{k} \leq \frac{1}{n} H .
$$

In [7] (cf. [8, Proposition 2.1]), it was shown that

$$
\left(\partial_{t}-\Delta\right) \bar{k} \leq\left(|A|^{2}+n K\right) \bar{k}-2 n K\left(\bar{k}-\frac{1}{n} H\right)
$$

and

$$
\left(\partial_{t}-\Delta\right) \underline{k} \geq\left(|A|^{2}+n K\right) \underline{k}+2 n K\left(\frac{1}{n} H-\underline{k}\right)
$$

in the viscosity sense along a solution to mean curvature flow.

By the calculations in 93.1 , the function

$$
F:=\sqrt{4 K+\frac{1}{n-2} H^{2}-|A|^{2}}
$$

is positive and satisfies

$$
\left(\partial_{t}-\Delta\right) F \geq\left(|A|^{2}+n K\right) F-(2 n-4) K F
$$

on a solution to mean curvature flow which initially satisfies the pinching condition (3.1). Thus, for such a solution,

$$
\begin{aligned}
\left(\partial_{t}-\Delta\right) \frac{\bar{k}}{F} & \leq-4 K\left(\frac{\bar{k}}{F}-\frac{1}{2} \frac{H}{F}\right)+2\left\langle\nabla \frac{\bar{k}}{F}, \nabla \log F\right\rangle \\
& \leq-4 K\left(\frac{\bar{k}}{F}-C\right)+2\left\langle\nabla \frac{\bar{k}}{F}, \nabla \log F\right\rangle
\end{aligned}
$$

in the viscosity sense, where

$$
C^{2}:=\frac{(n-2)(n-2+\alpha)}{4 \alpha} .
$$

Since the pinching condition is invariant under orientation reversal, the maximum principle then yields the following noncollapsing estimates.

Proposition 3.2. Let $X: \mathcal{M} \times[0, T) \rightarrow \mathbb{S}_{K}^{n+1}, n \geq 3$, be a solution to mean curvature flow such that $X_{0}: \mathcal{M} \rightarrow \mathbb{S}_{K}^{n+1}$ is embedded and satisfies (3.1) with $\alpha \in(0,1)$ when $n \geq 4$ or $\alpha \in\left(\frac{2}{3}, 1\right)$ if $n=3$. If

$$
\max _{\mathcal{M} \times\{0\}} \frac{\underline{k}}{\bar{F}} \geq-\mu \text { and } \max _{\mathcal{M} \times\{0\}} \frac{\bar{k}}{F} \leq \mu
$$

for some $\mu \geq C:=\sqrt{\frac{(n-2)(n-2+\alpha)}{4 \alpha}}$, then

$$
\frac{\underline{k}}{F}(p, t) \geq-C-(\mu-C) \mathrm{e}^{-4 K t} \text { and } \frac{\bar{k}}{F}(p, t) \leq C+(\mu-C) \mathrm{e}^{-4 K t}
$$


for all $(p, t) \in \mathcal{M} \times[0, T)$.

Corollary 3.3. For any solution $X: \mathcal{M} \times[0, T) \rightarrow \mathbb{S}_{K}^{n+1}$ to mean curvature flow in $\mathbb{S}_{K}^{n+1}, n \geq 3$, with embedded initial condition satisfying (3.1), there is a constant $\mu=\mu\left(n, \alpha, \min _{\mathcal{M} \times\{0\}} \frac{\underline{k}}{|H|+\sqrt{K}}, \max _{\mathcal{M} \times\{0\}} \frac{\bar{k}}{|H|+\sqrt{K}}\right)<\infty$ such that

$$
\underline{k} \geq-\mu(|H|+\sqrt{K}) \text { and } \bar{k} \leq \mu(|H|+\sqrt{K}) .
$$

3.4. The surgery class. Given $n \geq 3, K>0, \alpha \in(0,1), V<\infty$ and $\Theta<\infty$, we shall work with the class $\mathcal{C}_{K}^{n}(\alpha, V, \Theta)$ of hypersurfaces $X: \mathcal{M} \rightarrow \mathbb{S}_{K}^{n+1}$ satisfying

(1) $\max _{\mathcal{M}^{n} \times\{0\}}\left(|A|^{2}-\frac{1}{n-2+\alpha} H^{2}\right) \leq 2(2-\alpha) K^{2}$,

(2) $\mu_{0}\left(\mathcal{M}^{n}\right) \leq V K^{-\frac{n}{2}}$, and

(3) $\max _{\mathcal{M}^{n} \times\{0\}} H^{2} \leq \Theta K$,

where $\mu_{t}$ is the measure induced by $X(\cdot, t)$. Every properly immersed hypersurface of $\mathbb{S}_{K}^{n+1}$ which satisfies the strict quadratic pinching condition (1.2) lies in the class $\mathcal{C}_{K}^{n}(\alpha, V, \Theta)$ for some choice of parameters $\alpha, V$, and $\Theta$ (with $\alpha>\frac{2}{3}$ when $n=3$ ). The first two conditions are preserved under mean curvature flow; the third is not. However, it is possible to preserve the class $\mathcal{C}_{K}^{n}(\alpha, V, \Theta)$ under mean curvature flowwith-surgery in the following sense. Given an initial hypersurface in the class $\mathcal{C}_{K}^{n}(\alpha, V, \Theta)$, we will be able to choose $\Theta_{3}>\Theta_{2}>\Theta_{1}$ (depending only on $n, \alpha, V$ and $\Theta$ ) such that, if we stop the flow when $H^{2}$ reaches the threshold $\Theta_{3} K$, then regions of squared mean curvature at least $\Theta_{1} K$ form 'necks' of such quality that they may be replaced by high quality 'caps', resulting in a new hypersurface in the class $\mathcal{C}_{K}^{n}\left(\alpha, V, \Theta_{2}\right)$. Since the estimates pass, with the same constants, to flows modified by such surgeries, the procedure can be repeated with the same constants each time $H^{2}$ reaches the threshold $\Theta_{3} K$.

If, in addition, the initial datum $X_{0}: \mathcal{M} \rightarrow \mathbb{S}_{K}^{n+1}$ is an embedding, then we can also find $\mu>0$ such that the inscribed and exscribed curvatures are $\mu$-pinched, in the sense that

$$
\min _{\mathcal{M} \times\{0\}} \frac{\underline{k}}{|H|+\sqrt{K}} \geq-\mu \text { and } \max _{\mathcal{M} \times\{0\}} \frac{\bar{k}}{|H|+\sqrt{K}} \leq \mu .
$$

These inequalities are preserved under mean curvature flow in the sense of Corollary 3.3 .

Remark 3.4. The class $\mathcal{C}_{K}^{n}(\alpha, V, \Theta)$ is defined slightly differently than the class $\mathcal{C}\left(R, \alpha_{0}, \alpha_{1}, \alpha_{2}\right)$ of hypersurfaces of $\mathbb{R}^{n+1}$ introduced in [21]. 
We have found it convenient to use $K$ as the scale parameter here; formally, it is related to the scale parameter $R$ by the equation $R^{-2}=\Theta K$. The parameter $\alpha$ corresponds to $\alpha_{0}$ and $V$ corresponds to $\alpha_{3} \Theta^{\frac{n}{2}}$. The parameter $\alpha_{1}$ (a scale covariant lower bound for the mean curvature) is not required here - it is needed in [21] in order to bound the time of existence $T$ from above. Our estimates do not depend on a bound for $T$ and, indeed, some components of the flow may exist for all time.

\section{The Key EStimates FOR SMOOTH FLOWS}

4.1. The cylindrical estimate. The following estimate provides a suitable analogue of the Huisken-Sinestrari "cylindrical estimate" [21, Theorem 5.3].

Theorem 4.1 (Cylindrical estimate (Cf. [18,20,21,25])). Let X: $\mathcal{M} \times$ $[0, T) \rightarrow \mathbb{S}_{K}^{n+1}, n \geq 3$, be a solution to mean curvature flow with initial condition in the class $\mathcal{C}_{K}^{n}(\alpha, V, \Theta)$. Assume, further, that $\alpha>\frac{2}{3}$ when $n=3$. There exist $\delta=\delta(n, \alpha)>0, \eta_{0}=\eta_{0}(n, \alpha)>0$ and, for every $\eta \in\left(0, \eta_{0}\right), C_{\eta}=C_{\eta}(n, \alpha, V, \Theta, \eta)<\infty$ such that

$$
|A|^{2}-\frac{1}{n-1} H^{2} \leq \eta H^{2}+C_{\eta} K \mathrm{e}^{-2 \delta K t} \quad \text { in } \quad \mathcal{M}^{n} \times[0, T) .
$$

Remark 4.2. Note that, unlike the Euclidean analogue [21, Theorem 5.3], the constant $C_{\eta}$ does not depend on a bound for the maximal time (which is controlled by the minimum of $H$ at the initial time in [21]), and the zeroth order term becomes negligible for large times. This is because, as in [20, 2.1 Theorem], the coercive term in (4.2) gives rise to an exponential decay term in the " $L^{2}$-estimate", which we are able to exploit. This observation is useful here since, unlike in the Euclidean setting, solutions satisfying the initial conditions (1)-(3) can exist for all time (e.g. stationary hyperequators).

Remark 4.3. As a Corollary of the cylindrical estimate, we find that any minimal hypersurface of $\mathbb{S}_{K}^{n+1}, n \geq 3$, satisfying (1.2) must be totally geodesic. This is a special case of Simons' Theorem [28]. More generally, any hypersurface $X_{0}: \mathcal{M} \rightarrow \mathbb{S}_{K}^{n+1}$ satisfying (1.2) which flows for all time under mean curvature flow (e.g. if $X_{0}(\mathcal{M})$ is embedded and divides the area of $\mathbb{S}_{K}^{n+1}$ in two) must converge to a hyperequator.

We also find that $|A|^{2}-\frac{1}{n-1} H^{2} \leq 0$ for any uniformly quadratically pinched ancient solution $X: \mathcal{M} \times(-\infty, 0) \rightarrow \mathbb{S}_{K}^{n+1}$ with uniformly bounded area and curvature as $t \rightarrow-\infty$. A theorem of Huisken and Sinestrari [22, Theorem 6.1] then implies that $X: \mathcal{M} \times(-\infty, 0) \rightarrow \mathbb{S}_{K}^{n+1}$ is either a stationary hyperequator or a shrinking hyperparallel. 
Corollary 4.4. Let $X: \mathcal{M} \times(-\infty, \omega) \rightarrow \mathbb{S}_{K}^{n+1}$ be an ancient solution to mean curvature flow. If

$-\limsup _{t \rightarrow-\infty} \max _{\mathcal{M} \times\{t\}}\left(|A|^{2}-\frac{1}{n-2} H^{2}\right)<4 K$,

$-\lim \sup \mu_{t}(\mathcal{M})<\infty$, and

$$
t \rightarrow-\infty
$$

$-\limsup _{t \rightarrow-\infty} \max _{\mathcal{M} \times\{t\}} H^{2}<\infty$,

then $X: \mathcal{M} \times(-\infty, \omega) \rightarrow \mathbb{S}_{K}^{n+1}$ is either a stationary hyperequator or a shrinking hyperparallel.

Motivated by [20, 2.1 Theorem] and [21, Theorem 5.3], we will prove the estimate (4.1) by obtaining a bound for the function

$$
f_{\sigma, \eta}:=\left[|A|^{2}-\left(\frac{1}{n-1}+\eta\right) H^{2}\right] W^{\sigma-1}
$$

for some $\sigma \in(0,1)$ and any $\eta \in\left(0, \frac{(2-\alpha)(n-\alpha)}{2 n(n-1)(n-2+\alpha)}\right)$, where, setting

$$
a:=\frac{1}{n-2+\alpha}-\frac{1}{n-1}-\eta+\frac{\alpha}{2 n(n-1)} \text { and } b:=2(2-\alpha),
$$

the function $W$ is defined by

$$
W:=a H^{2}+b K \text {. }
$$

Observe that $W>0$ and $f_{\sigma, \eta} \leq W^{\sigma}$. The final term in the constant $a$ is chosen in order to obtain the good gradient and reaction terms in the following lemma.

Lemma 4.5. There exists $\delta=\delta(n, \alpha)>0$ such that

$$
\begin{aligned}
\left(\partial_{t}-\Delta\right) f_{\sigma, \eta} \leq & 2 \sigma\left(|A|^{2}+n K\right) f_{\sigma, \eta}-4 \delta K f_{\sigma, \eta} \\
& -2 \delta f_{\sigma, \eta} \frac{|\nabla A|^{2}}{W}+2(1-\sigma)\left\langle\nabla f_{\sigma, \eta}, \frac{\nabla W}{W}\right\rangle
\end{aligned}
$$

wherever $f_{\sigma, \eta}>0$.

Proof. Set $f_{\eta}:=|A|^{2}-\left(\frac{1}{n-1}+\eta\right) H^{2}$. Basic manipulations (independent of the precise form of $f_{\eta}$ and $W$ ) yield

$$
\begin{aligned}
\left(\partial_{t}-\Delta\right) f_{\sigma, \eta}= & W^{\sigma-1}\left(\partial_{t}-\Delta\right) f_{\eta}-(1-\sigma) f_{\sigma, \eta} W^{-1}\left(\partial_{t}-\Delta\right) W \\
& +2(1-\sigma)\left\langle\nabla f_{\sigma, \eta}, \frac{\nabla W}{W}\right\rangle-\sigma(1-\sigma) f_{\sigma, \eta} \frac{|\nabla W|^{2}}{W^{2}}
\end{aligned}
$$

The final term will be discarded.

Applying (2.12) and (2.11), we compute

$$
\left(\partial_{t}-\Delta\right) W=2\left(|A|^{2}+n K\right)(W-b K)-2 a|\nabla H|^{2}
$$


and

$$
\begin{aligned}
\left(\partial_{t}-\Delta\right) f_{\eta}= & 2\left(|A|^{2}+n K\right) f_{\eta}-4 n K\left(|A|^{2}-\frac{1}{n} H^{2}\right) \\
& -2\left(|\nabla A|^{2}-\left(\frac{1}{n-1}+\eta\right)|\nabla H|^{2}\right) .
\end{aligned}
$$

Combining the preceding three identities and estimating $f_{\sigma, \eta} \leq W^{\sigma}$, $|\nabla H|^{2} \leq \frac{n+2}{3}|\nabla A|^{2}$ and $\sigma<1$ yields

$$
\begin{aligned}
\left(\partial_{t}-\Delta\right) f_{\sigma, \eta} \leq & 2 \sigma\left(|A|^{2}+n K\right) f_{\sigma, \eta}+2(1-\sigma)\left\langle\nabla f_{\sigma, \eta}, \frac{\nabla W}{W}\right\rangle \\
& +2 W^{\sigma-1}\left(b K\left(|A|^{2}+n K\right) \frac{f_{\eta}}{W}-2 n K\left(|A|^{2}-\frac{1}{n} H^{2}\right)\right) \\
& -2 f_{\sigma, \eta}\left(1-\frac{n+2}{3}\left[\frac{1}{n-1}+\eta+a\right]\right) \frac{|\nabla A|^{2}}{W} .
\end{aligned}
$$

Consider the term

$$
\begin{aligned}
Z & :=(2-\alpha)\left(|A|^{2}+n K\right) \frac{f_{\eta}}{W}+H^{2}-n|A|^{2} \\
& \leq(2-\alpha)\left(\frac{1}{n-2+\alpha} H^{2}+b K+n K\right) \frac{f_{\eta}}{W}+H^{2}-n|A|^{2} .
\end{aligned}
$$

Noting that

$$
n a=(2-\alpha)\left(\frac{1}{n-2+\alpha}-\frac{1}{2(n-1)}\right)-n \eta,
$$

and estimating $f_{\eta} \leq W$, we find

$$
\begin{aligned}
Z & \leq n f_{\eta}+\left(\left[\frac{2-\alpha}{2(n-1)}+n \eta\right] H^{2}+\left[2-\alpha-\frac{n}{2}\right] b K\right) \frac{f_{\eta}}{W}+H^{2}-n|A|^{2} \\
& \leq-\left(\frac{1}{2(n-1)} H^{2}+\left[\alpha+\frac{n}{2}-2\right] b K\right) \frac{f_{\eta}}{W} .
\end{aligned}
$$

Now set

$$
\begin{aligned}
\delta & :=\min \left\{1-\frac{n+2}{3}\left[\frac{1}{n-2+\alpha}-\frac{\alpha}{2 n(n-1)}\right], \frac{a^{-1}}{2(n-1)}, \alpha+\frac{n}{2}-2\right\} \\
& >0 .
\end{aligned}
$$

We wish to bound $\mathrm{e}^{2 \delta K t} f_{\sigma, \eta}$ from above. It will suffice to consider points where $f_{\sigma, \eta}>0$. To that end, we consider the function

$$
f_{+}:=\max \left\{\mathrm{e}^{2 \delta K t} f_{\sigma, \eta}, 0\right\} .
$$


Lemma 4.6. There exist constants $\ell=\ell(n, \alpha, \eta)<\infty$ and $C=$ $C(n, K, \alpha, V, \Theta, \sigma, p)$ such that

$$
\int f_{+}^{p} d \mu \leq C \mathrm{e}^{-\delta p K t}
$$

so long as $\sigma \leq \ell p^{-\frac{1}{2}}$ and $p>\ell^{-1}$.

Proof. Applying (4.2) yields

$$
\begin{aligned}
\frac{d}{d t} \int f_{+}^{p} d \mu= & p \int f_{+}^{p-1} \partial_{t} f_{\sigma, \eta} d \mu-\int f_{+}^{p} H^{2} d \mu \\
\leq & -p(p-1) \int f_{+}^{p-2}\left|\nabla f_{\sigma, \eta}\right|^{2} d \mu-2 \delta p \int f_{+}^{p} \frac{|\nabla A|^{2}}{W} d \mu \\
& +2 \sigma p \int f_{+}^{p}|A|^{2} d \mu-2 \delta p K \int f_{+}^{p} d \mu \\
& +2 p \int f_{+}^{p} \frac{\left|\nabla f_{\sigma, \eta}\right|}{f_{\sigma, \eta}} \frac{|\nabla W|}{W} d \mu \\
\leq & -p\left(p-p^{\frac{1}{2}}-1\right) \int f_{+}^{p-2}\left|\nabla f_{\sigma, \eta}\right|^{2} d \mu \\
& -\left(2 \delta p-4 a n p^{\frac{1}{2}}\right) \int f_{+}^{p} \frac{|\nabla A|^{2}}{W} d \mu \\
& +2 \sigma p \int f_{+}^{p}|A|^{2} d \mu-2 \delta p K \int f_{+}^{p} d \mu .
\end{aligned}
$$

To estimate the penultimate term, we apply Proposition 2.2. Setting $u^{2}=f_{+}^{p}$ and $r=p^{\frac{1}{2}}$, this yields

$$
\begin{aligned}
2 \int f_{+}^{p}|A|^{2} d \mu & \leq C \int f_{+}^{p} W d \mu \\
& \leq C \int f_{+}^{p}\left(p^{\frac{3}{2}} \frac{\left|\nabla f_{\sigma, \eta}\right|}{f_{\sigma, \eta}^{2}}+p^{\frac{1}{2}} \frac{|\nabla A|^{2}}{W}+K\right) d \mu,
\end{aligned}
$$

where $C$ depends on $n, \alpha$ and $\eta$, and we thereby arrive at

$$
\begin{aligned}
\frac{d}{d t} \int f_{+}^{p} d \mu \leq & -p\left(p-C \sigma p^{\frac{3}{2}}-p^{\frac{1}{2}}-1\right) \int f_{+}^{p-2}\left|\nabla f_{\sigma, \eta}\right|^{2} d \mu \\
& -\left(2 \delta p-C \sigma p^{\frac{3}{2}}-4 a n p^{\frac{1}{2}}\right) \int f_{+}^{p} \frac{|\nabla A|^{2}}{W} d \mu \\
& -(2 \delta-C \sigma) p K \int f_{+}^{p} d \mu .
\end{aligned}
$$


Choosing $\sigma \leq \ell p^{-\frac{1}{2}}$ and $p \geq \ell^{-1}$, for $\ell=\ell(n, \alpha, \eta)$ sufficiently small, we can arrange that

$$
\frac{d}{d t} \log \left(\int f_{+}^{p} d \mu\right) \leq-\delta p K
$$

and hence

$$
\int f_{+}^{p} d \mu \leq \mathrm{e}^{-\delta p K t} \int f_{+}^{p}(\cdot, 0) d \mu_{0} \leq C \mathrm{e}^{-\delta p K t},
$$

where $C=C(n, K, \alpha, V, \Theta, \sigma, p)$.

This $L^{2}$-estimate (for $v:=f_{+}^{\frac{p}{2}}$ ) can be bootstraped to an $L^{\infty}$-estimate using Stampacchia iteration.

Proof of Theorem 4.1. Given $k \geq 0$, consider

$$
v_{k}^{2}:=\left(\mathrm{e}^{2 \delta K t} f_{\sigma, \eta}-k\right)_{+}^{p} \quad \text { and } \quad V_{k}(t):=\left\{x \in \mathcal{M}^{n}: v_{k}(x, t)>0\right\}
$$

and set

$$
u(k):=\int_{0}^{T} \int v_{k}^{2} d \mu d t \text { and } U(k):=\int_{0}^{T} \int_{V_{k}} d \mu d t .
$$

Note that, for any $h>k>0$,

$$
(h-k)^{p} U(h) \leq u(k) .
$$

So we need an estimate for $u(k)$. Computing as in (4.5), we can estimate

$$
\begin{aligned}
\frac{d}{d t} \int v_{k}^{2} d \mu+\int_{V_{k}}\left|\nabla v_{k}\right|^{2} d \mu+\int_{V_{k}} H^{2} v_{k}^{2} d \mu & \leq 2 \sigma p \int_{V_{k}} f_{+}^{p}\left(|A|^{2}+n K\right) d \mu \\
& \leq c_{n} \sigma p \int_{V_{k}} f_{+}^{p} W d \mu
\end{aligned}
$$

for any $k>0$ if $p>2$, where $c_{n}$ depends only on $n$.

We shall exploit the good gradient term using the Sobolev inequality (Theorem 2.3). Indeed, since (4.6) implies that

$$
\left|V_{k}\right| \leq k^{-p} \int f_{+}^{p} d \mu \leq C k^{-p},
$$

we can apply (2.20) to obtain

$$
\frac{1}{c_{n}}\left(\int v_{k}^{2^{*}} d \mu\right)^{\frac{1}{2^{*}}} \leq \int\left(\left|\nabla v_{k}\right|^{2}+H^{2} v_{k}^{2}\right) d \mu
$$

so long as

$$
k>k_{0}:=\left(\frac{(n+1) C}{\omega_{n}} K^{\frac{n}{2}}\right)^{p} .
$$


Recalling (4.8), we arrive at

$$
\frac{d}{d t} \int v_{k}^{2} d \mu+\left(\int v_{k}^{2^{*}} d \mu\right)^{\frac{1}{2^{*}}} \leq c_{n} \sigma p \int_{V_{k}} f_{+}^{p} W d \mu
$$

for $k>k_{0}$. Assuming that $k_{0}>\sup _{\mathcal{M} \times\{0\}} f_{\sigma, \eta}$, integration then yields

$$
\sup _{[0, T)} \int v_{k}^{2} d \mu+\int_{0}^{T}\left(\int v_{k}^{2^{*}} d \mu\right)^{\frac{1}{2^{*}}} \leq c_{n} \sigma p \int_{0}^{T} \int_{V_{k}} f_{+}^{p} W d \mu
$$

for $k>k_{0}$.

Using the interpolation inequality, we can estimate

$$
\int v_{k}^{\frac{2(n+2)}{n}} d \mu \leq\left(\int v_{k}^{2} d \mu\right)^{\frac{2}{n}}\left(\int v_{k}^{2^{*}} d \mu\right)^{\frac{2}{2^{*}}}
$$

so that, by Young's inequality,

$$
\begin{aligned}
\left(\int_{0}^{T} \int v_{k}^{\frac{2(n+2)}{n}} d \mu d t\right)^{\frac{n}{n+2}} & \leq\left(\sup _{t \in[0, T)} \int v_{k}^{2} d \mu\right)^{\frac{2}{n+2}}\left(\int_{0}^{T}\left(\int v_{k}^{2^{*}} d \mu\right)^{\frac{2}{2^{*}}} d t\right)^{\frac{n}{n+2}} \\
& \leq \frac{2}{n+2} \sup _{t \in[0, T)} \int v_{k}^{2} d \mu+\frac{n}{n+2} \int_{0}^{T}\left(\int v_{k}^{2^{*}} d \mu\right)^{\frac{2}{2^{*}}} d t \\
& \leq c_{n} \sigma p \int_{0}^{T} \int_{V_{k}} f_{+}^{p} W d \mu .
\end{aligned}
$$

Set $\sigma^{\prime}:=\sigma+\frac{1}{p} \lesssim p^{-\frac{1}{2}}$. Given $r>1$ (to be determined momentarily), we may choose $\ell$ slightly smaller if necessary, depending now also on $r$, so that Hölder's inequality and the $L^{2}$-estimate (4.6) yield

$$
\begin{aligned}
\int_{0}^{T} \int_{V_{k}} f_{+}^{p} W d \mu d t & \leq U(k)^{1-\frac{1}{r}}\left(\int_{0}^{T} \int_{V_{k}} f_{+}^{p r} W^{r} d \mu d t\right)^{\frac{1}{r}} \\
& =U(k)^{1-\frac{1}{r}}\left(\int_{0}^{T} \int_{V_{k}}\left(f_{\sigma^{\prime}, \eta}\right)_{+}^{p r} d \mu d t\right)^{\frac{1}{r}} \\
& \leq U(k)^{1-\frac{1}{r}}\left(C \int_{0}^{T} \mathrm{e}^{-\delta p r K t} d t\right)^{\frac{1}{r}} \\
& =\left[\frac{C}{\delta p r K}\left(1-\mathrm{e}^{-\delta p r K T}\right)\right]^{\frac{1}{r}} U(k)^{1-\frac{1}{r}}
\end{aligned}
$$

for $p \geq \ell^{-1}$ and $\sigma \leq \ell p^{-\frac{1}{2}}$, where $C=C(n, K, \alpha, V, \Theta, \sigma, p)$. 
Since, by Hölder's inequality,

$$
u(k) \leq U(k)^{\frac{2}{n+2}}\left(\int_{0}^{T} \int v_{k}^{\frac{2(n+2)}{n}} d \mu d t\right)^{\frac{n}{n+2}},
$$

the estimates (4.11) and (4.12) yield

$$
u(k) \leq C U(k)^{\frac{2}{n+2}+1-\frac{1}{r}}
$$

for $k>k_{0}$, where $C=C(n, K, \alpha, V, \Theta, \sigma, p, r)$. We conclude from (4.7) that

$$
(h-k)^{p} U(h) \leq C U(k)^{\gamma}
$$

where $\gamma:=1+\frac{2}{n+2}-\frac{1}{r}$ and $C=C(n, K, \alpha, V, \Theta, \sigma, p, r)$.

At this point, we fix $r>1+\frac{2}{n}$ (so that $\gamma>1$ ), $p^{-1}=\ell(n, \alpha, \eta)$, and $\sigma=\ell p^{-\frac{1}{2}}=\ell^{\frac{3}{2}}$. Stampacchia's Lemma [29, Lemma 4.1] then yields

$$
U\left(k_{0}+d\right)=0
$$

where

$$
d^{p}:=2^{p \gamma /(\gamma-1)} C U\left(k_{0}\right)^{\gamma-1} .
$$

Estimating via (4.6) (assuming $k_{0} \geq 1$ )

$$
U\left(k_{0}\right) \leq k_{0}^{-p} \int_{0}^{T} \int f_{+}^{p} d \mu d t \leq C(n, K, \alpha, V, \Theta, \eta),
$$

we conclude that

$$
\mathrm{e}^{2 \delta K t} f_{\sigma, \eta} \leq C(n, K, \alpha, V, \Theta, \eta)
$$

Young's inequality then yields

$$
|A|^{2}-\frac{1}{n-1} H^{2} \leq 2 \eta H+C(n, K, \alpha, V, \Theta, \eta) \mathrm{e}^{-2 \delta K t} .
$$

The theorem follows by the scaling covariance of the estimate.

4.2. The gradient estimate. Next, we derive a suitable analogue of the "gradient estimate" [21, Theorem 6.1]. We need the following a priori interior estimates for solutions with initial data in the class $\mathcal{C}_{K}^{n}(\alpha, V, \Theta)$.

Proposition 4.7. Let $X: \mathcal{M} \times[0, T) \rightarrow \mathbb{S}_{K}^{n+1}$ be a maximal solution to mean curvature flow with initial condition in the class $\mathcal{C}_{K}^{n}(\alpha, V, \Theta)$. Defining $\Lambda_{0}$ and $\lambda_{0}$ by

$$
\Lambda_{0} / 2:=\frac{1}{n-2+\alpha} \Theta^{2}+2(2-\alpha) \text { and } \mathrm{e}^{2 n \lambda_{0}}:=1+\frac{n}{n+\Lambda_{0}},
$$


we have

$$
\mathrm{e}^{2 n K T} \geq 1+\frac{2 n}{\Lambda_{0}}
$$

and

$$
\max _{\mathcal{M} \times\left\{\lambda_{0} K^{-1}\right\}}\left|\nabla^{k} A\right|^{2} \leq \Lambda_{k} K^{k+1}
$$

for every $k \in \mathbb{N}$, where $\Lambda_{k}$ depends only on $n, k$ and $\Lambda_{0}$.

Proof. Since

$$
\max _{\mathcal{M} \times\{0\}}|A|^{2} \leq \Lambda_{0} K / 2
$$

a straightforward ODE comparison argument applied to the inequality

$$
\left(\partial_{t}-\Delta\right)|A|^{2} \leq 2\left(|A|^{2}+n K\right)|A|^{2}
$$

yields

$$
\max _{\mathcal{M} \times\{t\}}|A|^{2} \leq \frac{n K}{\left(1+\frac{2 n}{\Lambda_{0}}\right) \mathrm{e}^{-2 n K t}-1} .
$$

We immediately obtain (4.15) and

$$
|A|^{2}(\cdot, t) \leq \Lambda_{0} K \text { for all } t \leq \lambda_{0} K^{-1} .
$$

The claim (4.16) now follows from the Bernstein estimates (Proposition 2.1).

Modifying an argument of Huisken [18, Theorem 6.1] and HuiskenSinestrari [21, Theorem 6.1], we can now obtain a pointwise estimate for the gradient of the second fundamental form which holds up to the singular time.

Theorem 4.8 (Gradient estimate (cf. [21, Theorem 6.1])). Let $X$ : $\mathcal{M} \times[0, T) \rightarrow \mathbb{S}_{K}^{n+1}, n \geq 2$, be a solution to mean curvature flow with initial condition in the class $\mathcal{C}_{K}^{n}(\alpha, V, \Theta)$. There exist constants $\delta=\delta(n, \alpha), c=c(n, \alpha, \Theta)<\infty, \eta_{0}=\eta_{0}(n)>0$ and, for every $\eta \in\left(0, \eta_{0}\right), C_{\eta}=C_{\eta}(n, \alpha, V, \Theta, \eta)<\infty$ such that

$$
|\nabla A|^{2} \leq c\left[\left(\eta+\frac{1}{n-1}\right) H^{2}-|A|^{2}\right] W+C_{\eta} K^{2} \mathrm{e}^{-2 \delta K t}
$$

in $\mathcal{M} \times\left[\lambda_{0} K^{-1}, T\right)$, where $\lambda_{0}$ is defined by (4.14).

Remark 4.9. As for the cylindrical estimate, the constants do not depend on a bound for the maximal time, and the zeroth order term becomes negligible for large times. 
Note that the conclusion is not vacuous since, by Proposition 4.7 . the maximal existence time of a solution with initial data in the class $\mathcal{C}_{K}^{n}(\alpha, V, \Theta)$ is at least $\frac{1}{2 n K} \log \left(1+\frac{2 n}{\Lambda_{0}}\right)>\lambda_{0} K^{-1}$.

Setting $\eta=1$, say, yields the cruder estimate

$$
|\nabla A|^{2} \leq C\left(H^{4}+K^{2}\right)
$$

where $C=C(n, \alpha, V, \Theta)$.

Proof of Theorem 4.8. We proceed as in [21, Theorem 6.1]. By (2.13),

$$
\left(\partial_{t}-\Delta\right)|\nabla A|^{2} \leq-2\left|\nabla^{2} A\right|^{2}+c_{n}\left(|A|^{2}+n K\right)|\nabla A|^{2} .
$$

We will control the bad term using the good term in the evolution equation for $|A|^{2}$ and the Kato inequality (2.4).

By the cylindrical estimate, given any $\eta>0$ we can find a constant $C_{\eta}=C_{\eta}(n, \alpha, V, \Theta, \eta)>2$ such that

$$
|A|^{2}-\frac{1}{n-1} H^{2} \leq \eta H^{2}+C_{\eta} K \mathrm{e}^{-2 \delta K t}
$$

and hence

$$
G_{\eta}:=2 C_{\eta} K \mathrm{e}^{-2 \delta K t}+\left(\eta+\frac{1}{n-1}\right) H^{2}-|A|^{2} \geq C_{\eta} K \mathrm{e}^{-2 \delta K t}>0 .
$$

Similarly, there is a constant $C_{0}=C_{0}(n, \alpha, V, \Theta)>2$ such that

$$
|A|^{2}-\frac{1}{n-1} H^{2} \leq \frac{2(n-2)}{(n+2)(n-1)} H^{2}+C_{0} K
$$

which ensures that

$$
G_{0}:=2 C_{0} K+\frac{3}{n+2} H^{2}-|A|^{2} \geq C_{0} K>0 .
$$

By (4.3),

$$
\begin{aligned}
\left(\partial_{t}-\Delta\right) G_{\eta}= & 2\left(|A|^{2}+n K\right)\left(G_{\eta}-2 C_{\eta} K \mathrm{e}^{-2 \delta K t}\right)+4 n K\left(|A|^{2}-\frac{1}{n} H^{2}\right) \\
& +2\left[|\nabla A|^{2}-\left(\eta+\frac{1}{n-1}\right)|\nabla H|^{2}\right]-2 \delta C_{\eta} K^{2} \mathrm{e}^{-2 \delta K t} .
\end{aligned}
$$

Since $G_{\eta} \geq C_{\eta} K \mathrm{e}^{-2 \delta K t}$, we can estimate $G_{\eta}-2 C_{\eta} K \mathrm{e}^{-2 \delta K t} \geq-G_{\eta}$. By the Kato inequality (2.4), we can estimate

$$
\begin{aligned}
|\nabla A|^{2}-\left(\frac{1}{n-1}+\eta\right)|\nabla H|^{2} & \geq \frac{n+2}{3}\left[\frac{3}{n+2}-\frac{1}{n-1}-\eta\right]|\nabla A|^{2} \\
& \geq \frac{\beta}{2}|\nabla A|^{2}
\end{aligned}
$$

where

$$
\beta:=\frac{1}{2}\left(\frac{3}{n+2}-\frac{1}{n-1}\right)
$$


so long as $\eta \leq\left(1-\frac{3}{4(n+2)}\right) \beta$. Estimating, finally,

$$
2 \delta C_{\eta} K^{2} \mathrm{e}^{-2 \delta K t} \leq 2 \delta K G_{\eta}
$$

we arrive at

$$
\left(\partial_{t}-\Delta\right) G_{\eta} \geq-2\left(|A|^{2}+n K\right) G_{\eta}+\beta|\nabla A|^{2}-2 \delta K G_{\eta} .
$$

Similarly,

$$
\left(\partial_{t}-\Delta\right) G_{0} \geq-2\left(|A|^{2}+n K\right) G_{0} .
$$

We seek a bound for the ratio $\frac{|\nabla A|^{2}}{G_{\eta} G_{0}}$. Note that, at a local spatial maximum of $\frac{|\nabla A|^{2}}{G_{\eta} G_{0}}$,

$$
0=\nabla_{k} \frac{|\nabla A|^{2}}{G_{\eta} G_{0}}=2 \frac{\left\langle\nabla_{k} \nabla A, \nabla A\right\rangle}{G_{\eta} G_{0}}-\frac{|\nabla A|^{2}}{G_{\eta} G_{0}}\left(\frac{\nabla_{k} G_{\eta}}{G_{\eta}}+\frac{\nabla_{k} G_{0}}{G_{0}}\right) .
$$

In particular,

$$
4 \frac{|\nabla A|^{2}}{G_{\eta} G_{0}}\left\langle\frac{\nabla G_{\eta}}{G_{\eta}}, \frac{\nabla G_{0}}{G_{0}}\right\rangle \leq \frac{|\nabla A|^{2}}{G_{\eta} G_{0}}\left|\frac{\nabla G_{\eta}}{G_{\eta}}+\frac{\nabla G_{0}}{G_{0}}\right|^{2} \leq 4 \frac{\left|\nabla^{2} A\right|^{2}}{G_{\eta} G_{0}} .
$$

Suppose that $\frac{|\nabla A|^{2}}{G_{\eta} G_{0}}$ attains a parabolic interior local maximum at $\left(x_{0}, t_{0}\right)$. Then, at $\left(x_{0}, t_{0}\right)$,

$$
\begin{aligned}
0 \leq & \left(\partial_{t}-\Delta\right) \frac{|\nabla A|^{2}}{G_{\eta} G_{0}} \\
= & \frac{\left(\partial_{t}-\Delta\right)|\nabla A|^{2}}{G_{\eta} G_{0}}-\frac{|\nabla A|^{2}}{G_{\eta} G_{0}}\left(\frac{\left(\partial_{t}-\Delta\right) G_{\eta}}{G_{\eta}}+\frac{\left(\partial_{t}-\Delta\right) G_{0}}{G_{0}}\right) \\
& +\frac{2}{G_{\eta} G_{0}}\left\langle\nabla \frac{|\nabla A|^{2}}{G_{\eta} G_{0}}, \nabla\left(G_{\eta} G_{0}\right)\right\rangle+2 \frac{|\nabla A|^{2}}{G_{\eta} G_{0}}\left\langle\frac{\nabla G_{\eta}}{G_{\eta}}, \frac{\nabla G_{0}}{G_{0}}\right\rangle \\
\leq & \frac{|\nabla A|^{2}}{G_{\eta} G_{0}}\left(\left(c_{n}+4\right)\left(|A|^{2}+n K\right)+2 \delta K-\beta \frac{|\nabla A|^{2}}{G_{\eta}}\right)
\end{aligned}
$$

and hence

$$
\frac{|\nabla A|^{2}}{G_{\eta} G_{0}} \leq \frac{\left(c_{n}+4\right)\left(|A|^{2}+n K\right)+2 \delta K}{2 C_{0} K+\frac{3}{n+2} H^{2}-|A|^{2}}
$$

Since

$$
|A|^{2} \leq \frac{1}{n-2+\alpha} H^{2}+2(2-\alpha) K,
$$

we obtain, at $\left(x_{0}, t_{0}\right)$,

$$
\frac{|\nabla A|^{2}}{G_{\eta} G_{0}} \leq C
$$

where $C$ depends only on $n$ and $\alpha$. 
On the other hand, since $G_{0}>C_{0} K$ and $G_{\eta}>C_{\eta} K \mathrm{e}^{-2 \delta K t}$, if no interior local parabolic maxima are attained, then, by Proposition 4.7, we have for any $t \geq \lambda_{0} K^{-1}$

$$
\begin{aligned}
\max _{\mathcal{M} \times\{t\}} \frac{|\nabla A|^{2}}{G_{0} G_{\eta}} & \leq \max _{\mathcal{M} \times\left\{\lambda_{0} K^{-1}\right\}} \frac{|\nabla A|^{2}}{G_{0} G_{\eta}} \\
& \leq \max _{\mathcal{M} \times\left\{\lambda_{0} K^{-1}\right\}} \frac{|\nabla A|^{2}}{C_{0} C_{\eta} K^{2} \mathrm{e}^{-2 \delta \lambda_{0}}} \\
& \leq \frac{\Lambda_{1} \mathrm{e}^{\lambda_{0}}}{C_{0} C_{\eta}} \\
& \leq \Lambda_{1} \mathrm{e}^{\lambda_{0}}
\end{aligned}
$$

The Theorem now follows from Young's inequality.

The following simple lemma illustrates the utility of scale-invariant, pointwise gradient estimates for the curvature.

Lemma 4.10. Let $X: \mathcal{M} \rightarrow \mathbb{S}^{n+1}$ be an immersed hypersurface. If

$$
\sup _{\mathcal{M}} \frac{|\nabla H|}{H^{2}} \leq c_{\sharp}<\infty
$$

then

$$
\frac{H(p)}{2} \leq H(q) \leq 2 H(p)
$$

for all $q \in \mathcal{B}_{\frac{1}{2 c_{\sharp} H(p)}}(p)$, the intrinsic ball of radius $\frac{1}{2 c_{\sharp} H(p)}$ about $p$.

Proof. For any unit speed geodesic $\gamma:[0, s] \rightarrow \mathcal{M}$ joining the points $y=\gamma(0)$ and $x=\gamma(s)$, we have

$$
\nabla_{\gamma^{\prime}} H^{-1} \leq c_{\sharp}
$$

Integrating yields

$$
-c_{\sharp} S \leq H^{-1}(x)-H^{-1}(y) \leq c_{\sharp} S
$$

or, if $s \leq \frac{1}{2 c_{\sharp} H(x)}$,

$$
\frac{H(x)}{2} \leq \frac{H(x)}{1+c_{\sharp} H(x) s} \leq H(y) \leq \frac{H(x)}{1-c_{\sharp} H(x) s} \leq 2 H(x) .
$$

The claim follows. 
4.3. Higher order estimates. The gradient estimate can be used to bound the first order terms which arise in the evolution equation for $\nabla^{2} A$. A straightforward maximum principle argument exploiting this observation yields an analagous estimate for $\nabla^{2} A$.

Theorem 4.11 (Hessian estimate (cf. [18,21])). Let $X: \mathcal{M} \times[0, T) \rightarrow$ $\mathbb{S}_{K}^{n+1}, n \geq 4$, be a solution to mean curvature flow with initial condition in the class $\mathcal{C}_{K}^{n}(\alpha, V, \Theta)$. There exists $C=C(n, \alpha, V, \Theta)$ such that

$$
\left|\nabla^{2} A\right|^{2} \leq C\left(H^{6}+K^{3}\right) \text { in } \mathcal{M} \times\left[\lambda_{0} K^{-1}, T\right) .
$$

Proof. We proceed as in [21, Theorem 6.3]. By (2.14),

$$
\left(\partial_{t}-\Delta\right)\left|\nabla^{2} A\right|^{2} \leq c\left(W\left|\nabla^{2} A\right|^{2}+|\nabla A|^{4}\right)-2\left|\nabla^{3} A\right|^{2},
$$

where $c$ depends only on $n$. It follows that

$$
\begin{aligned}
\left(\partial_{t}-\Delta\right) \frac{\left|\nabla^{2} A\right|^{2}}{W^{\frac{5}{2}}} \leq & \frac{c}{W^{\frac{5}{2}}}\left[W\left|\nabla^{2} A\right|^{2}+|\nabla A|^{4}\right]-2 \frac{\left|\nabla^{3} A\right|^{2}}{W^{\frac{5}{2}}} \\
& -5 a \frac{\left|\nabla^{2} A\right|^{2}}{W^{\frac{7}{2}}}\left[\left(|A|^{2}+n K\right) H^{2}-|\nabla H|^{2}\right] \\
& +\frac{5}{W^{\frac{7}{2}}}\left\langle\nabla\left|\nabla^{2} A\right|^{2}, \nabla W\right\rangle-\frac{25}{2} \frac{\left|\nabla^{2} A\right|^{2}}{W^{\frac{7}{2}}} \frac{|\nabla W|^{2}}{W} .
\end{aligned}
$$

We can use the good third order term on the first line to absorb the penultimate term, since

$$
\begin{aligned}
\frac{5}{W^{\frac{7}{2}}}\left\langle\nabla\left|\nabla^{2} A\right|^{2}, \nabla W\right\rangle & \leq \frac{10}{W^{\frac{7}{2}}}\left|\nabla^{3} A\right|\left|\nabla^{2} A\right||\nabla W| \\
& \leq \frac{1}{W^{\frac{1}{2}}}\left(\frac{\left|\nabla^{3} A\right|^{2}}{W^{2}}+25 \frac{\left|\nabla^{2} A\right|^{2}|\nabla W|^{2}}{W^{4}}\right) .
\end{aligned}
$$

Estimating

$$
\frac{|\nabla W|^{2}}{W} \leq 4 a|\nabla H|^{2}
$$

then yields

$$
\left(\partial_{t}-\Delta\right) \frac{\left|\nabla^{2} A\right|^{2}}{W^{\frac{5}{2}}} \leq \frac{c}{W^{\frac{5}{2}}}\left[W\left|\nabla^{2} A\right|^{2}+|\nabla A|^{4}\right]-\frac{\left|\nabla^{3} A\right|^{2}}{W^{\frac{5}{2}}}+55 a \frac{\left|\nabla^{2} A\right|^{2}}{W^{\frac{7}{2}}}|\nabla H|^{2} .
$$

Estimating the first order terms using Theorem 4.8 then yields

$$
\begin{aligned}
\left(\partial_{t}-\Delta\right) \frac{\left|\nabla^{2} A\right|^{2}}{W^{\frac{5}{2}} \leq} & c_{1} \frac{\left|\nabla^{2} A\right|^{2}}{W^{\frac{3}{2}}}+C_{1} K^{2} \frac{\left|\nabla^{2} A\right|^{2}}{W^{\frac{7}{2}}} \mathrm{e}^{-\delta K t} \\
& +\frac{c_{1} H^{4} W^{2}+C_{1} K^{4} \mathrm{e}^{-2 \delta K t}}{W^{\frac{5}{2}}}-\frac{\left|\nabla^{3} A\right|^{2}}{W^{\frac{5}{2}}}
\end{aligned}
$$

where $c_{1}$ depends only on $n, \alpha$ and $\Theta$, and $C_{1}$ depends also on $V$. 
Similar arguments yield

$$
\left(\partial_{t}-\Delta\right) \frac{|\nabla A|^{2}}{W^{\frac{3}{2}}} \leq \frac{c_{2} H^{2} W^{3}+C_{2} K^{4} \mathrm{e}^{-2 \delta K t}}{W^{\frac{5}{2}}}-\frac{\left|\nabla^{2} A\right|^{2}}{W^{\frac{3}{2}}},
$$

and

$$
\begin{aligned}
\left(\partial_{t}-\Delta\right) \frac{|\nabla A|^{2}}{W^{\frac{7}{2}}} & \leq c \frac{|\nabla A|^{2}}{W^{\frac{9}{2}}}\left(W^{2}+|\nabla H|^{2}\right)-\frac{\left|\nabla^{2} A\right|^{2}}{W^{\frac{7}{2}}} \\
& \leq \frac{c_{3} H^{2} W^{3}+C_{3} K^{4} \mathrm{e}^{-2 \delta K t}}{W^{\frac{9}{2}}}-\frac{\left|\nabla^{2} A\right|^{2}}{W^{\frac{7}{2}}}
\end{aligned}
$$

where $c_{2}$ and $c_{3}$ depend only on $n, \alpha$, and $\Theta$, and $C_{2}$ and $C_{3}$ depend also on $V$.

Setting

$$
f:=\frac{\left|\nabla^{2} A\right|^{2}}{W^{\frac{5}{2}}}+c_{1} \frac{|\nabla A|^{2}}{W^{\frac{3}{2}}}+C_{1} K^{2} \frac{|\nabla A|^{2}}{W^{\frac{7}{2}}}
$$

and estimating $W \geq K$, we obtain

$$
\begin{aligned}
\left(\partial_{t}-\Delta\right) f \leq & \frac{c_{1} H^{4} W^{2}+C_{1} K^{4} \mathrm{e}^{-2 \delta K t}}{W^{\frac{5}{2}}}+c_{1} \frac{c_{2} H^{2} W^{3}+C_{2} K^{4} \mathrm{e}^{-2 \delta K t}}{W^{\frac{5}{2}}} \\
& +C_{1} K^{2} \frac{c_{3} H^{2} W^{3}+C_{3} K^{4} \mathrm{e}^{-2 \delta K t}}{W^{\frac{9}{2}}} \\
\leq & \frac{\left(c_{1} a^{2}+c_{1} c_{2}+c_{3} C_{1}\right) H^{2} W^{3}+\left(C_{1}+c_{1} C_{2}+C_{1} C_{3}\right) K^{4} \mathrm{e}^{-2 \delta K t}}{W^{\frac{5}{2}}} \\
\leq & \left(c_{1} a^{2}+c_{1} c_{2}+c_{3} C_{1}\right) H^{2} W^{\frac{1}{2}}+\left(C_{1}+c_{1} C_{2}+C_{1} C_{3}\right) K^{\frac{3}{2}} \mathrm{e}^{-2 \delta K t} \\
\leq & c_{4}\left(|A|^{2}+n K\right) H+C_{4} K^{\frac{3}{2}} \mathrm{e}^{-2 \delta K t} .
\end{aligned}
$$

Thus,

$$
\left(\partial_{t}-\Delta\right)\left(f-c_{4} H+\frac{C_{4}}{2 \delta} K^{\frac{1}{2}} \mathrm{e}^{-2 \delta K t}\right) \leq 0
$$

The maximum principle and Proposition 4.7 then yield

$$
\begin{aligned}
\max _{\mathcal{M} \times\{t\}}\left(f-c_{4} H\right) & \leq \max _{\mathcal{M} \times\left\{\lambda_{0} K^{-1}\right\}}\left(f-c_{4} H\right)+\frac{C_{4}}{2 \delta} K^{\frac{1}{2}}\left(\mathrm{e}^{-2 \delta \lambda_{0}}-\mathrm{e}^{-2 \delta K t}\right) \\
& \leq C_{5} K^{\frac{1}{2}}
\end{aligned}
$$

for all $t \geq \lambda_{0} K^{-1}$, where $C_{5}$ depends only on $n, \alpha, V$, and $\Theta$. We conclude that

$$
\left|\nabla^{2} A\right|^{2} \leq c H W^{\frac{5}{2}}+C K^{\frac{1}{2}} W^{\frac{5}{2}} \text { in } \mathcal{M} \times\left[\lambda_{0} K^{-1}, T\right),
$$

where $c$ and $C$ depend only on $n, \alpha, V$, and $\Theta$. The claim now follows from Young's inequality. 
Applying the Hessian estimate in conjunction with the the evolution equation (2.9) for $A$ yields an analogous bound for $\nabla_{t} A$, and hence, in particular, for the time derivative of $H$. Thus, in high curvature regions, we obtain the following a priori bounds for $\nabla H$ and $\partial_{t} H$.

Corollary 4.12. Let $X: \mathcal{M} \times[0, T) \rightarrow \mathbb{S}_{K}^{n+1}, n \geq 2$, be a solution to mean curvature flow with initial condition in the class $\mathcal{C}_{K}^{n}(\alpha, V, \Theta)$. There exist $h_{\sharp}=h_{\sharp}(n, \alpha, V, \Theta)$ and $c_{\sharp}=c_{\sharp}(n, \alpha, V, \Theta)$ such that

$$
H(x, t) \geq h_{\sharp} \sqrt{K} \Longrightarrow \frac{|\nabla H|}{H^{2}}(x, t) \leq c_{\sharp} \text { and } \frac{\left|\partial_{t} H\right|}{H^{3}}(x, t) \leq \frac{c_{\sharp}^{2}}{2} .
$$

This is a very useful estimate in light of the following 'parabolic' version of Lemma 4.10.

Lemma 4.13. Let $X: \mathcal{M} \times[0, T) \rightarrow \mathbb{S}_{K}^{n+1}$ be a solution to mean curvature flow. If, given $c_{\sharp}<\infty$,

$$
\frac{|\nabla H|}{H^{2}} \leq c_{\sharp} \quad \text { and } \quad \frac{\left|\partial_{t} H\right|}{H^{3}} \leq \frac{c_{\sharp}^{2}}{2},
$$

then

$$
\frac{H(p, t)}{10} \leq H(q, s) \leq 10 H(p, t)
$$

for all $(q, s) \in \mathcal{P} \frac{1}{10 c_{\sharp} H(p, t)}(p, t)$, the intrinsic parabolic cylinder in $\mathcal{M} \times$ $[0, T)$ of radius $\frac{1}{10 c_{\sharp} H(p, t)}$ about $(p, t)$.

Proof. Fix $\gamma \in\left[\frac{1}{2}, 1\right)$. As in Lemma 4.10, given any $r \leq \frac{1-\gamma}{c_{\sharp} H(p, t)}$,

$$
\gamma H(p, t) \leq \frac{H(p, t)}{1+c_{\sharp} H(p, t) r} \leq H(q, t) \leq \frac{H(p, t)}{1-c_{\sharp} H(p, t) r} \leq \gamma^{-1} H(p, t)
$$

for all $q \in \mathcal{B}_{r}(p, t)$, the $g_{t}$-intrinsic ball of radius $r$ about the point $p$. Given $q \in \mathcal{B}_{r}(p, t)$, set $h(t):=H(p, t)$. Then

$$
-c_{\sharp}^{2} \leq\left(h^{-2}\right)^{\prime}(s) \leq c_{\sharp}^{2} .
$$

Since $r \leq \frac{1-\gamma}{c_{\sharp} H(p, t)} \leq \frac{\gamma}{c_{\sharp} H(p, t)} \leq \frac{1}{c_{\sharp} H(q, t)}$, integrating between $s \in\left(t-r^{2}, t\right]$ and $t$ yields

$$
\frac{H(q, t)}{\sqrt{1-c_{\sharp}^{2} H^{2}(q, t) r^{2}}} \leq H(q, s) \leq \frac{H(q, t)}{\sqrt{1-c_{\sharp}^{2} H^{2}(q, t) r^{2}}}
$$

and hence

$$
\frac{H(p, t)}{\sqrt{\gamma^{-2}+c_{\sharp}^{2} H^{2}(p, t) r^{2}}} \leq H(q, s) \leq \frac{H(p, t)}{\sqrt{\gamma^{2}-c_{\sharp}^{2} H^{2}(p, t) r^{2}}}
$$


for all $(q, s) \in \mathcal{P}_{r}(p, t)$, so long as $r \leq \frac{\gamma}{c_{\sharp} H(p, t)}$. The claim follows upon choosing, say, $\gamma=1 / 2$.

An inductive argument, exploiting estimates for lower order terms in the evolution equations for higher derivatives of $A$ as in Theorem 4.11, can be applied to obtain estimates for spatial derivatives of $A$ to all orders. The evolution equation for $A$ then yields bounds for the mixed space-time derivatives (cf. [21, Theorem 6.3 and Corollary 6.4]). We state these estimates here, however they will not actually be needed in the construction of the surgically modified flows.

Theorem 4.14 (Higher-order estimates). Let $X: \mathcal{M} \times[0, T) \rightarrow \mathbb{S}_{K}^{n+1}$, $n \geq 4$, be a solution to mean curvature flow with initial condition in the class $\mathcal{C}_{K}^{n}(\alpha, V, \Theta)$. There exist, for each pair of non-negative integers $k$ and $\ell$, constants $C_{k, \ell}=C_{k, \ell}(k, \ell, n, \alpha, V, \Theta)$ such that

$$
\left|\nabla_{t}^{k} \nabla^{\ell} A\right|^{2} \leq C_{k, \ell}\left(H^{2+4 k+2 \ell}+K^{1+2 k+\ell}\right) \text { in } \mathcal{M} \times\left[\lambda_{0} K^{-1}, T\right) .
$$

4.4. Neck detection. The cylindrical and gradient estimates imply that, in regions of very high curvature, solutions either form high quality 'neck' regions, or else become locally uniformly convex.

Lemma 4.15 (Curvature necks (cf. [21, Lemma 7.4])). Let $X: \mathcal{M} \times$ $[0, T) \rightarrow \mathbb{S}_{K}^{n+1}$ be a solution to mean curvature flow with initial condition in the class $\mathcal{C}_{K}^{n}(\alpha, V, \Theta)$. Given $\varepsilon \leq \frac{1}{100}$, there exist parameters $\eta_{\sharp}=\eta_{\sharp}(n, \alpha, V, \Theta, \varepsilon)>0$ and $h_{\sharp}=h_{\sharp}(n, \alpha, V, \Theta, \varepsilon)<\infty$ with the following property. If

$$
H\left(p_{0}, t_{0}\right) \geq h_{\sharp} \sqrt{K} \quad \text { and } \quad \lambda_{1}\left(p_{0}, t_{0}\right) \leq \eta_{\sharp} H\left(p_{0}, t_{0}\right),
$$

then

$$
\Lambda_{r_{0}, k, \varepsilon}\left(p_{0}, t_{0}\right) \leq \varepsilon r_{0}^{k+1}
$$

for each $k=0, \ldots,\left\lfloor\frac{2}{\varepsilon}\right\rfloor$, where $r_{0}:=\frac{n-1}{H\left(p_{0}, t_{0}\right)}$,

$$
\Lambda_{r, 0, \varepsilon}(p, t):=\max _{\mathcal{B}_{\varepsilon^{-1} r_{r}}(p, t) \times\left(t-10^{4} r^{2}, t\right]} \sqrt{\lambda_{1}^{2}+\sum_{j=2}^{n}\left(\lambda_{n}-\lambda_{j}\right)^{2}},
$$

and, for each $k \geq 1$,

$$
\Lambda_{r, k, \varepsilon}(p, t):=\max _{\mathcal{B}_{\varepsilon^{-1}}(p, t) \times\left(t-10^{4} r^{2}, t\right]}\left|\nabla^{k} A\right| .
$$

Proof. The proof is essentially that of [21, Lemma 7.4].

Suppose that the claim does not hold. Then for some $n \geq 3$ there must exist parameters $\alpha, V$ and $\Theta$, some $\varepsilon_{0}<\frac{1}{100}$, a sequence of 
solutions $X_{j}: \mathcal{M}_{j} \times\left[0, T_{j}\right) \rightarrow \mathbb{S}_{K_{j}}^{n+1}$ to mean curvature flow with $X_{j}(\cdot, 0) \in \mathcal{C}_{K_{j}}^{n}(\alpha, V, \Theta)$, and points $\left(p_{j}, t_{j}\right) \in \mathcal{M}_{j}^{n} \times\left[0, T_{j}\right)$ such that

$$
H_{j}\left(p_{j}, t_{j}\right) \geq(n-1) j \sqrt{K_{j}} \text { and } \frac{\lambda_{1}^{j}}{H_{j}}\left(p_{j}, t_{j}\right) \leq j^{-1} \text {, }
$$

and yet

$$
\Lambda_{r_{j}, k_{j}, \varepsilon_{0}}^{j}\left(p_{j}, t_{j}\right) \geq \varepsilon_{0} r_{j}^{k+1}
$$

for some $k_{j} \leq\left\lfloor\varepsilon_{0}^{-1}\right\rfloor$ for each $j$, where $r_{j}:=\frac{n-1}{H_{j}\left(x_{j}, t_{j}\right)}$ and we denote objects defined along $X_{j}$ using the a sub- or superscript $j$. After passing to a subsequence, we may arrange that (4.26) holds for some fixed $k_{j}=k_{0} \leq\left\lfloor\varepsilon_{0}^{-1}\right\rfloor$ for all $j$. After translating the points $\left(X_{j}\left(x_{j}, t_{j}\right), t_{j}\right)$ to the space-time origin in $\mathbb{R}^{n+2} \times \mathbb{R}$ and rotating so that the tangent plane to the sphere at the origin is $\mathbb{R}^{n+1} \times\{0\}$ with upward pointing normal, and parabolically rescaling by $r_{j}$, we obtain a sequence of flows

$$
\hat{X}_{j}: \mathcal{M}_{j}^{n} \times\left[-r_{j}^{-2} t_{j}, r_{j}^{-2}\left(T_{j}-t_{j}\right)\right) \rightarrow \mathbb{S}_{r_{j}^{2} K_{j}}^{n+1}-r_{j}^{-1} K_{j}^{-\frac{1}{2}} e_{n+2}
$$

given by

$$
\hat{X}_{j}(x, t):=r_{j}^{-1} O_{j}\left(X_{j}\left(x, r_{j}^{2} t+t_{j}\right)-X_{j}\left(x_{j}, t_{j}\right)\right),
$$

where $O_{j} \in \mathrm{SO}(n+1)$. Each $\hat{X}_{j}$ is in the class $\mathcal{C}_{r_{j}^{2} K_{j}}^{n}(\alpha, V, \Theta)$ and satisfies $\hat{X}_{j}\left(x_{j}, 0\right)=0$,

$$
\hat{H}_{j}\left(x_{j}, 0\right)=n-1, \text { and } \frac{\hat{\lambda}_{1}^{j}}{\hat{H}_{j}}\left(x_{j}, 0\right) \leq j^{-1},
$$

but

$$
\hat{\Lambda}_{1, k_{0}, \varepsilon_{0}}^{j}\left(x_{j}, 0\right) \geq \varepsilon_{0},
$$

where we denote objects defined along $\hat{X}_{j}$ using a $(\hat{\cdot})$ and the sub- or superscript $j$. We claim that the new sequence subconverges locally uniformly in the smooth topology to a shrinking cylinder solution in the Euclidean space $\mathbb{R}^{n+1} \times\{0\}$, in contradiction with (4.29). First note that, by (4.15), $K_{j} t_{j} \geq C(n, \alpha, \Theta)>0$ and hence $-r_{j}^{-2} t_{j} \rightarrow-\infty$ as $j \rightarrow \infty$. We claim that the mean curvature of $\hat{X}_{j}$ is uniformly bounded on an intrinsic parabolic cylinder of uniform radius about $\left(x_{j}, 0\right)$, so long as $j$ is sufficiently large. Indeed, by Theorems 4.8 and 4.11, we can find constants $c_{\sharp}$ (depending only on $n, \alpha$ and $\Theta$ ) and $C$ (depending only on $n, \alpha, V$ and $\Theta$ ) such that

$$
\left|\hat{\nabla}_{j} \hat{H}_{j}\right| \leq \frac{c_{\sharp}}{2} \hat{H}_{j}^{2}+C j^{-2} \text { and }\left|\partial_{t} \hat{H}_{j}\right| \leq \frac{c_{\sharp}^{2}}{4} \hat{H}_{j}^{3}+C j^{-3}
$$


in $\mathcal{M}_{j} \times\left[-r_{j}^{-2} t_{j}+j^{-2} / 4,0\right]$. Thus, given any $\rho>0$, we can find $j_{0} \in \mathbb{N}$ such that

$$
\left|\hat{\nabla}_{j} \hat{H}_{j}\right| \leq c_{\sharp} \hat{H}_{j}^{2} \text { and }\left|\partial_{t} \hat{H}_{j}\right| \leq \frac{c_{\sharp}^{2}}{2} \hat{H}_{j}^{3}
$$

in $\mathcal{M}_{j}^{n} \times[-\rho, 0]$ for $j \geq j_{0}$. Lemma 4.13 now implies that

$$
\frac{n-1}{10}=\frac{\hat{H}_{j}\left(x_{j}, 0\right)}{10} \leq \hat{H}_{j}(y, s) \leq 10 \hat{H}_{j}\left(x_{j}, 0\right)=10(n-1)
$$

for any $(y, s) \in \mathcal{P}_{\frac{1}{10 c_{\sharp}}}^{j}\left(x_{j}, 0\right)$ for all sufficiently large $j$. It follows that some subsequence of the restricted mean curvature flows $\left.\hat{X}_{j}\right|_{\mathcal{P}_{\frac{1}{j}}{ }_{10 c_{\sharp}}}\left(x_{j}, 0\right)$ converges locally uniformly in the smooth topology to a limiting mean curvature flow $\hat{X}: U \times\left(-\frac{1}{100 c_{\sharp}^{2}}, 0\right] \rightarrow \mathbb{R}^{n+1} \times\{0\}$ (which may not be proper). We claim that the limit flow is part of a shrinking cylinder. We shall denote objects defined along the limit using a $(\hat{\cdot})$. Indeed, by the cylindrical estimate (Theorem 4.1), $\hat{X}$ satisfies

$$
|\hat{A}|^{2}-\frac{1}{n-1} \hat{H}^{2} \leq 0 \text {. }
$$

In particular, $\hat{\lambda}_{1}$ is non-negative. On the other hand, by (4.28), $\hat{\lambda}_{1}$ vanishes at the origin. Thus, by the splitting theorem, $\hat{X}$ splits locally off a line. But then (4.30) implies that the cross section of the splitting is umbilic. We need to extend the convergence to a sufficiently large region. This can be achieved since, a posteriori, the mean curvature could not have increased very much in $\mathcal{P}_{\frac{1}{10 c_{\sharp}}}^{j}$ (due to the convergence to a shrinking cylinder solution). That is,

$$
\hat{H}_{j}(y, s) \leq 2(n-1)
$$

for all $(y, s) \in \mathcal{P}_{\frac{1}{10 c_{\sharp}}}\left(x_{j}, 0\right)$ so long as $j$ is sufficiently large. Applying the gradient estimates as before, we obtain uniform bounds for $\hat{H}_{j}$ on the uniformly larger neighborhood $\frac{\mathcal{P}_{\frac{2}{10 c_{\sharp}}}^{j}}{{ }_{0}}\left(x_{j}, 0\right)$. Repeating the previous argument, we conclude that a subsequence of the flows $\left.\hat{X}_{j}\right|_{\mathcal{P}^{j}{ }_{\frac{2}{10} \sharp}}\left(x_{j}, 0\right)$ converge to a part of shrinking cylinder. After repeating the argument a finite number of times, we obtain convergence of a subsequence of the flows $\left.\hat{X}_{j}\right|_{\mathcal{P}_{2 \varepsilon_{0}^{j}}^{j}\left(x_{j}, 0\right)}$ to a part of a shrinking cylinder. Since the convergence is smooth on compact subsets of spacetime, this violates (4.29). 
Definition 4.16. Let $X: \mathcal{M} \rightarrow \mathbb{S}_{K}^{n+1} \subset \mathbb{R}^{n+2}$ be an immersed hypersurface of $\mathbb{S}_{K}^{n+1}$. A point $p \in \mathcal{M}$ lies at the center of an $(\varepsilon, k, L)$-neck of size $r$ if the map $\exp _{r^{-1} X(p)}^{-1} \circ\left(r^{-1} X\right)$ is $\varepsilon$-cylindrical and $(\varepsilon, k)$-parallel at all points in the induced intrinsic ball of radius $L$ about $p$ in the sense of [21, Definition 3.9].

By [21, Propositions 3.4 and 3.5], these "curvature" necks can be integrated to obtain "hypersurface" necks in the tangent space, which can be replaced by a pair of "convex caps" in a controlled way (see [21, Section 3]).

\section{THE KEY ESTIMATES FOR SURGICALLY MODIFIED FLOWS}

We need to show that suitable versions of the key estimates still hold in the presence of surgeries. In the following definition, surgery is performed on the middle third of a neck of size $r$ in the obvious way:

(i) First scale by $r^{-1}$ and precompose with $\exp _{r^{-1} X(p)}^{-1}$ to obtain a neck in $T_{r^{-1} X(p)} \mathbb{S}_{r^{2} K}^{n+1}$

(ii) Perform the surgery on the middle third of this neck in $T_{r^{-1} X(p)} \mathbb{S}_{r^{2} K}^{n+1}$ as described in [21, Section 3],

(iii) Re-embed in $\mathbb{S}_{K}^{n+1}$ by composing with $\exp _{r^{-1} X(p)}$ and scaling by $r$.

Definition 5.1. A surgically modified (mean curvature) flow in $\mathbb{S}_{K}^{n+1}$ with neck parameters $(\varepsilon, k, L)$, surgery parameters $(\tau, B)$, and surgery scale $r$ is a finite sequence $\left\{X_{i}: \mathcal{M}_{i}^{n} \times\left[T_{i}, T_{i+1}\right] \rightarrow \mathbb{S}_{K}^{n+1}\right\}_{i=1}^{N-1}$ of smooth mean curvature flows $X_{i}: \mathcal{M}_{i}^{n} \times\left[T_{i}, T_{i+1}\right] \rightarrow \mathbb{S}_{K}^{n+1}$ for which the $(i+1)$ st initial datum $X_{i+1}\left(\cdot, T_{i+1}\right): \mathcal{M}_{i+1} \rightarrow \mathbb{S}_{K}^{n+1}$ is obtained from the $i$-th final datum $X_{i}\left(\cdot, T_{i+1}\right): \mathcal{M}_{i} \rightarrow \mathbb{S}_{K}^{n+1}$ by performing finitely many $(\tau, B)$-standard surgeries, in the sense of [21, Section 3], on the middle thirds of $(\varepsilon, k, L)$-necks with mean curvature satisfying $\frac{n-1}{10 r} \leq H \leq$ $\frac{10(n-1)}{r}$, and then discarding finitely many connected components that are diffeomorphic either to $\mathbb{S}^{n}$ or to $\mathbb{S}^{1} \times \mathbb{S}^{n-1}$.

5.1. Quadratic and inscribed/exscribed curvature pinching. For a suitable range of neck and surgery parameters, and surgery scales, the surgery procedure of [21, Section 3] preserves the quadratic pinching condition (1.2). Indeed, the surgery replaces a nearly cylindrical Euclidean neck satisfying $|A|^{2} \simeq \frac{1}{n-1} H^{2}$ with a pair of Euclidean convex caps satisfying $|A|^{2} \simeq \frac{1}{n} H^{2}$ (these estimates are carried out precisely in [26, Corollary 3.20]). Since the surgery scale may be taken arbitrarily small, the same can be ensured after re-embedding in $\mathbb{S}_{K}^{n+1}$. 
When $X_{0}: \mathcal{M} \rightarrow \mathbb{S}_{K}^{n+1}$ is an embedding, we can also preserve the inscribed curvature pinching

$$
\max _{\mathcal{M} \times\{0\}} \frac{\bar{k}}{\bar{F}} \leq \mu_{0}
$$

for any constant $\mu_{0} \geq \sqrt{\frac{(n-2)(n-2+\alpha)}{4 \alpha}}$. Indeed, by Proposition 3.2, $\max _{\mathcal{M} \times\{0\}} \frac{\bar{k}}{F}$ does not decay between surgeries. Moreover, using [21, Theorem 3.26], we can arrange, for suitable neck and surgery parameters, and surgery scales, that

$$
\frac{\bar{k}}{F} \leq \sqrt{\frac{(n-2)(n-2+\alpha)}{4 \alpha}}
$$

on the regions modified or added by surgery. A similar argument applies to the exscribed curvature.

5.2. The cylindrical estimate. We first note that the function $\left(f_{\sigma, \eta}\right)_{+}$ is pointwise non-increasing in regions modified by surgery.

Lemma 5.2. Given $n \geq 3$ and $K>0$, there exist parameters $\eta_{0}>0$, $\sigma_{0} \in(0,1)$, neck parameters $\varepsilon_{0}>0, k_{0} \geq 2$, surgery parameters $\tau, B$, and a surgery scale $r_{0}>0$ such that, for any $\sigma \in\left(0, \sigma_{0}\right]$ and $\eta \in\left(0, \eta_{0}\right]$, the function $\left(f_{\sigma, \eta}\right)_{+}$is

- zero on regions added by, and

- non-increasing on regions modified by

standard surgery with parameters $\tau_{0}, B$ on an $(\varepsilon, k, L)$-neck with mean curvature satisfying $H \geq \frac{(n-1)}{10 r}$ for any $\varepsilon \in\left(0, \varepsilon_{0}\right], k \geq k_{0}, L \geq 10$ and $r \in\left(0, r_{0}\right]$.

Proof. This follows readily from [21, Proposition 4.5] and the Gauss equation.

In the following theorem, we assume that the parameters $\eta, \sigma$, the neck parameters $\varepsilon, k, L$, the surgery parameters $\tau, B$, and the surgery scale $r$ are chosen within the range for which Lemma 5.2 applies.

Theorem 5.3 (Cylindrical estimate for surgically modified flows (Cf. [21, Theorem 5.3])). Let $\left\{X_{i}: \mathcal{M}_{i}^{n} \times\left[T_{i}, T_{i+1}\right] \rightarrow \mathbb{S}_{K}^{n+1}\right\}_{i=1}^{N-1}, n \geq 3$, be a surgically modified flow with initial condition in the class $\mathcal{C}_{K}^{n}(\alpha, V, \Theta)$ (with $\alpha>\frac{2}{3}$ when $\left.n=3\right)$. For every $\eta \in\left(0, \eta_{0}\right)$ there exists $C_{\eta}=$ $C_{\eta}(n, \alpha, V, \Theta, \eta)<\infty$ such that

$$
|A|^{2}-\frac{1}{n-1} H^{2} \leq \eta H^{2}+C_{\eta} K \quad \text { in } \quad \mathcal{M}_{i} \times\left[T_{i}, T_{i+1}\right]
$$

for all $i$. 
Proof. Proceeding as in the proof of Theorem 4.1 but with $\delta$ taken to be zero, we obtain an analogue of (4.9) on each time interval $\left(T_{i}, T_{i+1}\right)$, with $v_{k}$ replaced by $\left(f_{\sigma, \eta}-k\right)_{+}^{\frac{p}{2}}$. By Lemma 5.2 , this can be integrated from $T_{1}=0$ to $T_{N}=T$ to obtain an analogue of (4.10). The remainder of the proof of the cylindrical estimate then applies unmodified.

Henceforth, when we refer to a surgically modified flow, we will assume that the neck and surgery parameters, and the surgery scale, are fixed within a suitable range, which we progressively refine.

5.3. The gradient estimate. Since the derivatives of the second fundamental form are zero on round Euclidean cylinders and spherical caps, the derivative estimates also pass to surgically modified flows.

Theorem 5.4 (Gradient estimate for surgically modified flows (Cf. [21, Theorem 6.1])). Let $\left\{X_{i}: \mathcal{M}_{i}^{n} \times\left[T_{i}, T_{i+1}\right] \rightarrow \mathbb{S}_{K}^{n+1}\right\}_{i=1}^{N-1}, n \geq 3$, be a surgically modified flow with initial condition in the class $\mathcal{C}_{K}^{n}(\alpha, V, \Theta)$ (with $\alpha>\frac{2}{3}$ when $n=3$ ). There exists $C=C(n, \alpha, V, \Theta)<\infty$ such that

$$
|\nabla A|^{2} \leq C\left(H^{4}+K^{2}\right) \quad \text { in } \quad \mathcal{M}_{i}^{n} \times\left[T_{i}, T_{i+1}\right]
$$

for all $i$.

Proof. We proceed as in the proof of Theorem 4.8, but with $\delta$ taken to be zero and fixed $\eta=\beta$, where $\beta$ is defined by (4.20). First observe that, since $|A|^{2}-\frac{1}{n-1} H^{2} \equiv 0$ on a round cylinder in Euclidean space, we may choose a suitable range of neck and surgery parameters, and surgery scales, so that

$$
|A|^{2}-\frac{1}{n-1} H^{2} \leq \frac{\beta}{2} H^{2}
$$

on regions modified or added by surgery. We may therefore arrange that

$$
G_{\beta}:=\left(\frac{1}{n-1}+\beta\right) H^{2}-|A|^{2}+2 C_{\beta} K \geq \frac{\beta}{2} H^{2}
$$

and

$$
G_{0}:=\frac{3}{n+2} H^{2}-|A|^{2}+2 C_{0} K \geq \frac{3 \beta}{2} H^{2} .
$$

Furthermore, since $|\nabla A|^{2} \equiv 0$ on a round cylinder in Euclidean space, we may choose a suitable range of neck and surgery parameters, and surgery scales, so that, on regions modified or added by surgery, $|\nabla A|^{2} \leq$ 
$\mu_{0} H^{4}$, where $\mu_{0}$ is a constant which depends only on $n$. Thus, in regions modified or added by surgery,

$$
\frac{|\nabla A|^{2}}{G_{0} G_{\beta}} \leq \frac{4 \mu_{0}}{3 \beta^{2}}
$$

Since the surgically modified flow remains in a fixed surgery class, we may proceed as in the proof of Theorem 4.8 in the time intervals $\left(T_{i}, T_{i+1}\right)$.

5.4. Higher order estimates. Proceeding similarly as in Theorem 5.4 (cf. [21, Theorem 6.3]) yields estimates for higher derivatives of $A$ along surgically modified flows.

Theorem 5.5 (Hessian estimate for surgically modified flows (cf. [21, Theorem 6.3])). Let $\left\{X_{i}: \mathcal{M}_{i}^{n} \times\left[T_{i}, T_{i+1}\right] \rightarrow \mathbb{S}_{K}^{n+1}\right\}_{i=1}^{N-1}, n \geq 3$, be a surgically modified flow with initial condition in the class $\mathcal{C}_{K}^{n}(\alpha, V, \Theta)$ (with $\alpha>\frac{2}{3}$ when $n=3$ ). There exists $C=C(n, \alpha, V, \Theta)$ such that

$$
\left|\nabla^{2} A\right|^{2} \leq C\left(H^{6}+K^{3}\right) \text { in } \mathcal{M} \times\left[\lambda_{0} K^{-1}, T\right) .
$$

Proof. Proceed as in Theorem 4.11 between surgeries and use the fact that, for suitable neck and surgery parameters, and surgery scales, $\left|\nabla^{2} A\right|^{2} / H^{6}$ is small in regions modified or added by surgery.

Analogues of the higher order estimates (4.25) also pass to surgically modified flows, but, as mentioned above, they will not actually be needed in the construction.

5.5. Neck detection. The conclusion of the neck detection lemma 4.15 also holds for surgically modified flows, so long as we work in regions which are not affected by surgeries (cf. [21, Lemma 7.4]).

In the following theorem, a region $U \times I$ is free of surgeries if at each surgery time $T_{i} \in I, i \in\{2, \ldots, N-1\}$, we have $U \subset \mathcal{M}_{i-1} \cap \mathcal{M}_{i}$ and $\left.X_{i-1}\right|_{U}\left(\cdot, T_{i}\right)=\left.X_{i}\right|_{U}\left(\cdot, T_{i}\right)$ (and hence $X_{i-1}$ and $X_{i}$ may be pasted together to form a smooth mean curvature flow in $U \times I)$.

Theorem 5.6 (Neck detection for surgically modified flows (cf. [21, Lemma 7.4])). Let $\left\{X_{i}: \mathcal{M}_{i}^{n} \times\left[T_{i}, T_{i+1}\right] \rightarrow \mathbb{S}_{K}^{n+1}\right\}_{i=1}^{N-1}, n \geq 3$, be a surgically modified flow with initial condition in the class $\mathcal{C}_{K}^{n}(\alpha, V, \Theta)$. Given $\varepsilon, \theta, L$ and $k$, there exist positive $\eta_{\sharp}$, $h_{\sharp}$ with the following property: If

(ND1) $\left|H\left(p_{0}, t_{0}\right)\right| \geq h_{\sharp} \sqrt{K}$ and $\frac{\lambda_{1}\left(p_{0}, t_{0}\right)}{\left|H\left(p_{0}, t_{0}\right)\right|} \leq \eta_{\sharp}$, and

(ND2) the neighbourhood $\mathcal{P}\left(p_{0}, t_{0}, \frac{(n-1)(L+1)}{H\left(p_{0}, t_{0}\right)}, \frac{\theta}{H^{2}\left(p_{0}, t_{0}\right)}\right)$ is free of surgeries, 
then $\left(p_{0}, t_{0}\right)$ lies at the centre of an $(\varepsilon, k, L)$-neck.

Proof. The proof of Lemma 4.15applies using Theorems 5.3, 5.4 and5.5 in lieu of Theorems 4.1, 4.8 and 4.11, due to the hypothesis (ND2).

\section{EXISTENCE OF TERMINATING SURGICALLY MODIFIED FLOWS}

We say that a surgically modified flow $\left\{X_{i}: \mathcal{M}_{i}^{n} \times\left[T_{i}, T_{i+1}\right] \rightarrow\right.$ $\left.\mathbb{S}_{K}^{n+1}\right\}_{i=1}^{N-1}$ terminates at the final time $T:=T_{N}<\infty$ if either

- each connected component of $X_{N-1}\left(\mathcal{M}_{N-1}, T_{N}\right)$ is diffeomorphic to $\mathbb{S}^{n}$ or to $\mathbb{S}^{1} \times \mathbb{S}^{n-1}$, or

- after performing surgery on $X_{N-1}\left(\mathcal{M}_{N-1}, T_{N}\right)$, each connected component of the resulting hypersurface is diffeomorphic to $\mathbb{S}^{n}$ or to $\mathbb{S}^{1} \times \mathbb{S}^{n-1}$.

Theorem 6.1 (Existence of terminating surgically modified flows). Let $X: \mathcal{M} \rightarrow \mathbb{S}_{K}^{n+1}, n \geq 3$, be a properly immersed hypersurface satisfying the quadratic pinching condition (1.2). There exists a surgically modified flow $\left\{X_{i}: \mathcal{M}_{i}^{n} \times\left[T_{i}, T_{i+1}\right] \rightarrow \mathbb{S}_{K}^{n+1}\right\}_{i=1}^{N-1}$ with $X_{1}(\cdot, 0)=X$ which terminates at time $T=T_{N}$.

Proof. Given the cylindrical and gradient estimates, and the neck detection lemma, and a sufficiently small choice of the surgery scale $r$, we can proceed as in [21, Section 8] using the machinery developed in [21, Sections 3 and 7], with only minor modifications required. These are:

1. In order to reconcile our data $\mathcal{C}_{K}^{n}(\alpha, V, \Theta)$ with those of [21], we replace the parameter $K$ by introducing the scale factor $R:=1 / \sqrt{\Theta K}$. Our data $\alpha$ and $V$ can then be related to their $\alpha_{0}$ and $\alpha_{2}$, respectively. The constant $\alpha_{1}$ which appears in [21] is not needed here. Since the surgery scale may be taken as small as needed, we may then choose the surgery parameters (albeit with slightly worse values) as explained in [21, pp. 208-209].

2. Since our ambient space is non-Euclidean, the proof of the neck continuation theorem requires modification in two places. These are explained and carried out in detail in a more general setting in [10, Section 8].

3. Since the maximal time is not a priori bounded in the present setting, the surgery algorithm may not terminate "on its own". Observe, however, that the maximum of the mean curvature must eventually drop permanently below the scale which triggers the surgery; indeed, if this were not the case, then an infinite number of surgeries would be carried out, an impossibility since we began with a finite amount of area, area is non-increasing under the flow, and each surgery decreases 
area by at least a certain fixed amount. The flow can then be smoothly continued indefinitely. Since the curvature remains uniformly bounded, standard arguments imply that each connected component converges, along some sequence of times approaching infinity, to a minimal hypersurface. The cylindrical estimate (Theorem 4.1) applied independently to each connected component then implies that each component of the limit is a totally geodesic hypersphere. So the flow must terminate afterall.

\section{REFERENCES}

[1] Hilário Alencar and Manfredo do Carmo. Hypersurfaces with constant mean curvature in spheres. Proc. Amer. Math. Soc., 120(4):1223-1229, 1994.

[2] B. Andrews. Positively curved surfaces in the three-sphere. In Proceedings of the International Congress of Mathematicians, Vol. II (Beijing, 2002), pages 221-230. Higher Ed. Press, Beijing, 2002.

[3] Ben Andrews. Contraction of convex hypersurfaces in Riemannian spaces. $J$. Differential Geom., 39(2):407-431, 1994.

[4] Ben Andrews. Noncollapsing in mean-convex mean curvature flow. Geom. Topol., 16(3):1413-1418, 2012.

[5] Ben Andrews and Charles Baker. Mean curvature flow of pinched submanifolds to spheres. J. Differential Geom., 85(3):357-395, 2010.

[6] Ben Andrews, Bennett Chow, Christine Guenther, and Mat Langford. Extrinsic Geometric Flows. Graduate Studies in Mathematics, Vol. 206. American Mathematical Society, 2020.

[7] Ben Andrews, Xiaoli Han, Haizhong Li, and Yong Wei. Non-collapsing for hypersurface flows in the sphere and hyperbolic space. Ann. Sc. Norm. Super. Pisa Cl. Sci. (5), 14(1):331-338, 2015.

[8] Ben Andrews and Mat Langford. Two-sided non-collapsing curvature flows. Ann. Sc. Norm. Super. Pisa Cl. Sci. (5), 15:543-560, 2016.

[9] Ben Andrews, Mat Langford, and James McCoy. Non-collapsing in fully nonlinear curvature flows. Ann. Inst. H. Poincaré Anal. Non Linéaire, 30(1):23-32, 2013.

[10] Simon Brendle and Gerhard Huisken. A fully nonlinear flow for two-convex hypersurfaces in Riemannian manifolds. Invent. Math., 210(2):559-613, 2017.

[11] Reto Buzano, Robert Haslhofer, and Or Hershkovits. The moduli space of twoconvex embedded tori. Int. Math. Res. Not. IMRN, 2019(2):392-406, 2019.

[12] Reto Buzano, Robert Haslhofer, and Or Hershkovits. The moduli space of two-convex embedded spheres. Preprint, arXiv:1607.05604, 2020.

[13] Qing Ming Cheng and Hisao Nakagawa. Totally umbilic hypersurfaces. Hiroshima Math. J., 20(1):1-10, 1990.

[14] S. S. Chern, M. do Carmo, and S. Kobayashi. Minimal submanifolds of a sphere with second fundamental form of constant length. In Functional Analysis and Related Fields (Proc. Conf. for M. Stone, Univ. Chicago, Chicago, Ill., 1968), pages 59-75. Springer, New York, 1970.

[15] Robert Haslhofer and Bruce Kleiner. Mean curvature flow of mean convex hypersurfaces. Comm. Pure Appl. Math., 70(3):511-546, 2017. 
[16] Robert Haslhofer and Bruce Kleiner. Mean curvature flow with surgery. Duke Math. J., 166(9):1591-1626, 2017.

[17] David Hoffman and Joel Spruck. Sobolev and isoperimetric inequalities for Riemannian submanifolds. Commun. Pure Appl. Math., 27:715-727, 1974.

[18] Gerhard Huisken. Flow by mean curvature of convex surfaces into spheres. $J$. Differential Geom., 20(1):237-266, 1984.

[19] Gerhard Huisken. Contracting convex hypersurfaces in Riemannian manifolds by their mean curvature. Invent. Math., 84(3):463-480, 1986.

[20] Gerhard Huisken. Deforming hypersurfaces of the sphere by their mean curvature. Math. Z., 195(2):205-219, 1987.

[21] Gerhard Huisken and Carlo Sinestrari. Mean curvature flow with surgeries of two-convex hypersurfaces. Invent. Math., 175(1):137-221, 2009.

[22] Gerhard Huisken and Carlo Sinestrari. Convex ancient solutions of the mean curvature flow. J. Differential Geom., 101(2):267-287, 2015.

[23] J. H. Michael and L. M. Simon. Sobolev and mean-value inequalities on generalized submanifolds of $R^{n}$. Comm. Pure Appl. Math., 26:361-379, 1973.

[24] Alexander Mramor. A finiteness theorem via the mean curvature flow with surgery. J. Geom. Anal., 28(4):3348-3372, 2018.

[25] Huy The Nguyen. Convexity and cylindrical estimates for mean curvature flow in the sphere. Trans. Am. Math. Soc., 367(7):4517-4536, 2015.

[26] Huy The Nguyen. High codimension mean curvature flow with surgery. arXiv:2004.07163, 2020.

[27] Masafumi Okumura. Hypersurfaces and a pinching problem on the second fundamental tensor. Amer. J. Math., 96:207-213, 1974.

[28] James Simons. Minimal varieties in riemannian manifolds. Ann. of Math. (2), 88:62-105, 1968.

[29] Guido Stampacchia. Èquations elliptiques du second ordre à coefficients discontinus. Séminaire de Mathématiques Supérieures, No. 16 (Été, 1965). Les Presses de l'Université de Montréal, Montreal, Que., 1966.

Department of Mathematics, University of Tennessee Knoxville, KNOXVILLE TN, USA, 37996-1320

School of Mathematical and Physical Sciences, The University of Newcastle, Newcastle, NSW, Australia, 2308

E-mail address: mlangford@utk.edu, mathew.langford@newcastle.edu.au

School of Mathematical Sciences, Queen Mary University of LonDon, Mile End RoAd, London, UK, E1 4NS

E-mail address: h.nguyen@qmul.ac.uk 